\title{
Monitoring local delivery of vancomycin from gelatin nanospheres in zebrafish larvae
}

This article was published in the following Dove Press journal:

International Journal of Nanomedicine

\author{
Xiaolin Zhang ${ }^{1,2, *}$ \\ Jiankang Song,* \\ Alexey Klymov ${ }^{3, *}$ \\ Yang Zhang ${ }^{3}$ \\ Leonie de Boer' \\ John $\mathrm{A}$ Jansen ${ }^{3}$ \\ Jeroen JJP van den Beucken ${ }^{3}$ \\ Fang Yang ${ }^{3}$ \\ Sebastian AJ Zaat ${ }^{1, *}$ \\ Sander CG \\ Leeuwenburgh ${ }^{3, *}$ \\ 'Department of Medical Microbiology, \\ Amsterdam Infection and Immunity \\ Institute, Academic Medical Center, \\ University of Amsterdam, Amsterdam, \\ the Netherlands; ' ${ }^{2}$ Department of \\ Biomaterials Science and Technology, \\ MIRA Institute for Biomedical \\ Technology and Technical Medicine, \\ University of Twente, Enschede, \\ the Netherlands; ${ }^{3}$ Department of \\ Biomaterials, Radboud University \\ Medical Centre, Nijmegen, \\ the Netherlands \\ *These authors contributed equally \\ to this work
}

\begin{abstract}
Background: Infections such as biomaterial-associated infection and osteomyelitis are often associated with intracellular survival of bacteria (eg, Staphylococcus aureus). Treatment of these infections remains a major challenge due to the low intracellular efficacy of many antibiotics. Therefore, local delivery systems are urgently required to improve the therapeutic efficacy of antibiotics by enabling their intracellular delivery.

Purpose: To assess the potential of gelatin nanospheres as carriers for local delivery of vancomycin into macrophages of zebrafish larvae in vivo and into THP-1-derived macrophages in vitro using fluorescence microscopy.
\end{abstract}

Materials and methods: Fluorescently labeled gelatin nanospheres were prepared and injected into transgenic zebrafish larvae with fluorescent macrophages. Both the biodistribution of gelatin nanospheres in zebrafish larvae and the co-localization of vancomycin-loaded gelatin nanospheres with zebrafish macrophages in vivo and uptake by THP-1-derived macrophages in vitro were studied. In addition, the effect of treatment with vancomycin-loaded gelatin nanospheres on survival of $S$. aureus-infected zebrafish larvae was investigated.

Results: Internalization of vancomycin-loaded gelatin nanospheres by macrophages was observed qualitatively both in vivo and in vitro. Systemically delivered vancomycin, on the other hand, was hardly internalized by macrophages without the use of gelatin nanospheres. Treatment with a single dose of vancomycin-loaded gelatin nanospheres delayed the mortality of S. aureus-infected zebrafish larvae, indicating the improved therapeutic efficacy of vancomycin against (intracellular) $S$. aureus infection in vivo.

Conclusion: The present study demonstrates that gelatin nanospheres can be used to facilitate local and intracellular delivery of vancomycin.

Keywords: in vivo real-time monitoring, fluorescence microscopy, biodistribution, cell-material interaction, Staphylococcus aureus, intracellular infection

\section{Introduction}

Bacteria such as Staphylococcus aureus (S. aureus) are responsible for various infectious diseases such as pneumonia, bacteremia, diabetic foot infection, osteomyelitis, endocarditis, and biomaterial-associated infection. ${ }^{1-4}$ Such infections are often chronic and difficult to treat since $S$. aureus is a facultative intracellular pathogen, and even host phagocytes often fail to kill these intracellular bacteria. ${ }^{5-8}$ Although some antibiotics (eg, quinolones) are effective to treat intracellular infection, unfortunately, many antibiotics and other antimicrobial agents (eg, macrolides, aminoglycosides, oxazolidinone, $\beta$-lactams, glycopeptides, ansamycin, and antimicrobial peptides) have limited antibacterial activity against intracellular bacteria due to low cell penetration rates, low intracellular retention, or development of resistance. ${ }^{9,10}$ Therefore, several types of nanocarriers (eg, liposomes, nanoparticles, dendrimers, and polymersomes)
Correspondence: Sander CG Leeuwenburgh

Department of Biomaterials, Radboud University Medical Center, PO Box 9101, $6500 \mathrm{HB}$ Nijmegen, the Netherlands

$\mathrm{Tel}+31615409006$

Fax +3I 243614657

Email sander.leeuwenburgh@

radboudumc.nl
International Journal of Nanomedicine 2018:I 3 5377-5394

Dovepress if in 1

http://dx.doi.org/10.21471!N.S168959 (c) (7) (5) 2018 Zhang et al. This work is published and licensed by Dove Medical Press Limited. The full terms of this license are available at https://www.dovepress.com/terms.php cC. hereby accept the Terms. Non-commercial uses of the work are permitted without any further permission from Dove Medical Press Limited, provided the work is properly attributed. For permission for commercial use of this work, please see paragraphs 4.2 and 5 of our Terms (https://www.dovepress.com/terms.php). 
have been designed to improve local delivery of antibiotics and enhance their efficacy against intracellular bacterial infections. ${ }^{11,12}$ Different raw materials such as natural polymers (eg, collagen ${ }^{13}$ and alginate ${ }^{14}$ ) and synthetic polymers (eg, poly (lactide-co-glycolide) [PLGA] ${ }^{15}$ and poly (amino acids) $)^{16}$ have been used to develop these delivery systems, but none of them is optimal yet. For instance, collagen and alginate are relatively expensive and often contain impurities, ${ }^{11}$ while several synthetic polymers such as PLGA are degraded to acidic products reducing the $\mathrm{pH}$ in the local/ systemic microenvironment which may affect cell function negatively. ${ }^{17}$ Therefore, only few formulations based on polymeric micro/nanoparticles have been approved for market introduction. ${ }^{12}$ More importantly, no delivery systems are available yet, which support complete eradication of intracellular bacteria. ${ }^{11}$

Gelatin nanospheres are promising carriers for local delivery of antibiotics based on their inherent advantages. First of all, gelatin has been widely used for biomedical research owing to its biocompatibility, biodegradability, and cost-effectiveness. ${ }^{18-21}$ Second, the charged nature of gelatin nanospheres facilitates controlled delivery and release of oppositely charged macromolecules such as growth factors $^{22-24}$ and nucleic acids ${ }^{25,26}$ as well as small biomolecules such as vancomycin and colistin. ${ }^{27-29}$ Third, the cross-linking density of gelatin nanospheres can be simply adjusted to tune the degradation rate of gelatin nanospheres. ${ }^{22,24,27,30}$ Finally, the potential of gelatin nanospheres as carriers for local delivery of biomolecules was confirmed previously in vitro. ${ }^{26,31}$ This potential was, however, not yet confirmed in vivo due to lack of suitable animal models. In addition, the spatial distribution of gelatin nanospheres in vivo is largely unknown, even though this biodistribution strongly determines the final applicability of gelatin nanospheres as carriers for site-specific delivery of drugs. Hence, in vivo assessment of the biodistribution of gelatin nanospheres as well as their potential for local delivery of antibiotics is highly desired to further the development of gelatin nanosphere-based delivery systems for infection treatment.

Zebrafish larvae have been used as in vivo models to study infection pathogenesis ${ }^{32,33}$ and assess the therapeutic efficacy of drug-delivery systems. ${ }^{34,35}$ Advantages of the use of zebrafish larvae include the following: strong resemblance of their immune system to the human immune system, their high fecundity as well as their external fertilization, rapid development, and easy maintenance. ${ }^{36-38}$ These features render zebrafish larvae a cost- and space-effective animal model with the potential for medium- to high-throughput analyses for in vivo research. ${ }^{32,39}$ Moreover, the optical transparency of zebrafish larvae enables real-time visualization and intravital analysis of cellular responses to pathogens or materials. . $^{34,37,40}$ To this end, a variety of transgenic zebrafish lines expressing different fluorescent proteins in different types of cells (eg, macrophages and neutrophils) has been developed. ${ }^{37}$

The aim of the present study was to evaluate the cellular response to gelatin nanospheres in vivo in zebrafish larvae and assess the potential of gelatin nanospheres as carriers to allow for internalization of vancomycin by macrophages, as studied in live larvae using fluorescence microscopy. To this end, we labeled gelatin nanospheres and vancomycin with fluorescence to study 1) the biodistribution of gelatin nanospheres and 2) the internalization of vancomycin by fluorescent macrophages of zebrafish larvae (in vivo) as well as THP-1-derived macrophages (in vitro). Vancomycin was selected as model antibiotic since it has limited antibacterial activity inside cells. ${ }^{7,94}$ In addition, we compared the effect of systemic versus local delivery of vancomycin (from gelatin nanospheres) on survival of $S$. aureus-infected zebrafish larvae. We observed in a qualitative manner that vancomycin was internalized by macrophages upon delivery from gelatin nanospheres both in vitro and in vivo. Moreover, a single dose of local delivery of vancomycin from gelatin nanospheres improved the survival of $S$. aureus-infected zebrafish larvae, which warrants further in vivo pharmacokinetic studies in a quantitative manner in an alternative in vivo model.

\section{Materials and methods Materials}

Gelatin B was obtained from Sigma-Aldrich (Zwijndrecht, the Netherlands) with Bloom number of 225 and an isoelectric point of $\sim 5$. A $25 \mathrm{wt} \%$ glutaraldehyde solution (in water) was obtained from Acros Organics (Geel, Belgium). DyLight $^{\mathrm{TM}} 650$ NHS Esters and BODIPY-FL ${ }^{\circledR}$-conjugated vancomycin (designated as fluorescent-labeled vancomycin in this manuscript) were obtained from ThermoFisher Scientific (Bleiswijk, the Netherlands). Vancomycin hydrochloride and all other chemicals used in this study were obtained from Sigma-Aldrich. The zebrafish medium (E3 medium) was prepared according to a standard protocol. ${ }^{42}$

\section{Experimental setup}

The experimental setup in the present study is schematically depicted in Figure S1.

\section{Preparation of gelatin nanospheres}

Gelatin nanospheres were prepared via a two-step coacervation method, as previously described in detail. ${ }^{27,43}$ In brief, 
gelatin $(1.25 \mathrm{~g})$ was dissolved in Milli-Q water $(25 \mathrm{~mL})$ at $50^{\circ} \mathrm{C}$ under magnetic stirring to form a $5 \%(\mathrm{w} / \mathrm{v})$ solution. High molecular weight chains of gelatin were precipitated by adding acetone $(25 \mathrm{~mL})$. After discarding the supernatant, Milli-Q water $(25 \mathrm{~mL})$ was added to redissolve the gelatin at $50^{\circ} \mathrm{C}$ under magnetic stirring, followed by adjusting the $\mathrm{pH}$ of the gelatin solution to 2.5 with $1 \mathrm{M} \mathrm{HCl}$. Acetone $(80 \mathrm{~mL})$ was subsequently added $(4 \mathrm{~mL} / \mathrm{min})$ under highspeed $(1,200 \mathrm{rpm})$ stirring to allow formation of gelatin nanospheres. Subsequently, the gelatin nanospheres were cross-linked using glutaraldehyde $(25 \mathrm{wt} \%, 740 \mu \mathrm{L})$. After this overnight cross-linking reaction, glycine solution (100 mM, $105 \mathrm{~mL}$ ) was added to terminate the cross-linking process. The gelatin nanospheres were washed in Milli-Q and collected via 3 cycles of washing and centrifuging (5,000 rpm, 60 minutes). The suspension of gelatin nanospheres in Milli-Q $(18 \mathrm{mg} / \mathrm{mL})$ was kept at $4^{\circ} \mathrm{C}$ for further use. The gelatin nanopsheres are stable in Milli-Q at least for 3 months due to the absence of proteolytic enzymes.

\section{Fluorescent labeling of gelatin nanospheres}

To track gelatin nanospheres in the zebrafish larvae, gelatin nanospheres were fluorescent-labeled with a far red dye (DyLight $^{\text {TM }} 650$ NHS esters) (designated as fluorescently labeled gelatin nanospheres) following the manufacturer's protocol. In brief, gelatin nanospheres $(200 \mathrm{mg})$ were dispersed in phosphate buffered saline (PBS, $20 \mathrm{~mL}$ ). Sodium hydroxide solution $(1 \mathrm{M})$ was then added to adjust the $\mathrm{pH}$ of the gelatin nanosphere suspension to 7.2. Subsequently, NHS ester solution (50 $\mu \mathrm{g}$ in $200 \mu \mathrm{L}$ of N,N-dimethylformamide) was added to the gelatin nanosphere suspension to react for 1 hour under magnetic stirring (400 rpm). The fluorescently labeled gelatin nanospheres were subsequently centrifuged and washed using Milli-Q water (5,000 rpm, 60 minutes). The suspension of fluorescently labeled gelatin nanospheres $(18 \mathrm{mg} / \mathrm{mL})$ was stored at $4{ }^{\circ} \mathrm{C}$ for further use.

\section{Characterization of gelatin nanospheres}

The unlabeled and labeled gelatin nanospheres were sputtercoated with gold, and the morphology of these gelatin nanospheres was examined using scanning electron microscopy (SEM; LEO 435 VP; Zeiss, Jena, Germany; Sigma-300, Zeiss). The zeta potential of the gelatin nanospheres was characterized by laser Doppler electrophoresis using a Zetasizer Nano-Z instrument (Malvern Instruments Ltd., Worcestershire, UK) in PBS buffer and Milli-Q water. The size of the gelatin nanospheres in swollen state was examined in $154 \mathrm{mM}$ PBS at $\mathrm{pH} 7.4$ and Milli-Q water at $\mathrm{pH} 7.0$ by dynamic light scattering (DLS; Zetasizer Nano-S, Malvern Instruments Ltd., Worcestershire, UK).

\section{Zebrafish husbandry and maintenance of larvae}

The zebrafish larvae used for studies using fluorescent microcopy were from the transgenic lines (mpeg1:Gal4/ UAS:Kaede) and (fms:Gal4/UAS:mCherry), which express green and red fluorescent proteins, respectively, in their macrophages. ${ }^{44,45}$ The larvae used for assessing treatment efficacy of $S$. aureus infection (see paragraph below) were from the wild-type Tupfel long fin (TL). The husbandry and maintenance of adult zebrafish followed the welfare regulations of animals approved by the local animal welfare committee (Dierexperimentencommissie, DEC) and were described in detail before. ${ }^{40,43}$ After harvesting, zebrafish larvae were maintained in E3 medium as described. ${ }^{40,43}$

\section{Microinjection of gelatin nanospheres into zebrafish larvae}

At 3 days post-fertilization, zebrafish larvae were anesthetized by using $0.03 \%$ tricaine (buffered 3 aminobenzoic acid ethyl ester; Sigma-Aldrich), placed in agarose plates (with U-shaped grooves, home-made) containing E3 medium. Gelatin nanosphere suspension was vortexed for 1 minute and subsequently loaded into a glass capillary (Harvard Apparatus, Holliston, MA, USA) which was connected to an Eppendorf FemtoJet microinjector (Nijmegen, the Netherlands). The size of the capillary tip was adjusted to around $15 \mu \mathrm{m}$ in diameter by breaking manually under a Leica M20 light microscope (Eindhoven, the Netherlands). This allowed injection of a volume of $\sim 3 \mathrm{~nL}$. To study the distribution of gelatin nanospheres and macrophagegelatin nanospheres interactions, the suspension of gelatin nanospheres was injected either into the blood circulation of zebrafish larvae via the Duct of Cuvier ${ }^{46}$ or into the tail muscle tissue, ${ }^{40,46}$ under a light microscope.

\section{Visualization of biodistribution of and macrophage response to gelatin nanospheres injected into zebrafish larvae} We visualized the distribution of and the response of macrophages to the injected gelatin nanospheres in zebrafish larvae of the transgenic line (mpeg1:Gal4/UAS:Kaede) at 3 and 24 hours post-injection by using confocal laser scanning microscopy (Leica TCS SP8 SMD). The zebrafish larvae were anesthetized as described earlier and placed in a $35 \mathrm{~mm}$ glass-bottom culture dish (MatTek Corporation, 
Ashland, MA, USA). The larvae were subsequently covered with $1.5 \mathrm{wt} \%$ low melting point agarose. The entire sample was then covered by E3 medium after solidification of the agarose. A series of Z-stack images were recorded and subsequently converted to three-dimensional (3D) videos using the Leica LAS X software. Figures explained in the results section and their corresponding $3 \mathrm{D}$ videos were in all cases recorded from one representative larva in each experimental group. Macrophages of zebrafish larvae of the transgenic line (mpeg1:Gal4/UAS:Kaede) express Kaede fluorescent protein. This fluorescent protein may undergo an irreversible color change from green to red upon UV illumination (350-400 nm), depending on the exposure time and energy level. ${ }^{47,48}$ However, in control experiments, this color conversion was not observed under the current experimental conditions (Figure S2).

\section{Visualization of internalization of gelatin nanospheres by THP-I-derived macrophages}

THP-1-derived macrophages were cultured in medium containing gelatin nanospheres. Internalization of gelatin nanospheres by these macrophages in vitro was studied, as previously described in detail. ${ }^{43}$ In brief, THP-1 human monocytic cells were seeded onto Thermanox coverslips (ThermoFisher Scientific) at a density of $2.5 \times 10^{5}$ cells $/ \mathrm{cm}^{2}$ in a 24 -well plate containing $500 \mu \mathrm{L}$ of RPMI 1640 medium $\left(\right.$ Gibco $^{\mathrm{TM}}$, ThermoFisher Scientific, Paisley, Scotland). The medium was supplemented with fetal bovine serum $(10 \%)$, streptomycin $(100 \mu \mathrm{g} / \mathrm{mL})$, and penicillin $(100 \mathrm{U} / \mathrm{mL})$. The cells were cultured at $37^{\circ} \mathrm{C}$ and under $5 \% \mathrm{CO}_{2}$. Phorbol-12-myristate-13-acetate $(50 \mathrm{ng} / \mathrm{mL}$, PMA; Sigma-Aldrich) was added to the culture medium to induce differentiation into macrophages. After culturing for 48 hours, fluorescently labeled gelatin nanospheres in Milli-Q water $(5 \mu \mathrm{L}, 18 \mathrm{mg} / \mathrm{mL})$ were added to the plate and the THP1-derived macrophages were incubated with these gelatin nanospheres for 24 hours. After incubation, Cell-Tracker ${ }^{\mathrm{TM}}$ CMFDA green (10 $\mu \mathrm{M}$; ThermoFisher Scientific) was added to stain the cell cytoplasm. After an additional incubation for 30 minutes, the cells were washed with PBS for 3 times and fixed with 4\% paraformaldehyde. The nucleic acid of THP-1derived macrophages was stained with DAPI (Roche Applied Science, Mannheim, Germany), and subsequently mounted with ProLong ${ }^{\circledR}$ Diamond Antifade Mountant (ThermoFisher Scientific) for imaging using confocal microscopy. A series of Z-stack images were recorded and subsequently converted to $3 \mathrm{D}$ videos using the Leica LAS X software.

\section{Visualization of internalization of vancomycin-loaded gelatin nanospheres by THP-I-derived macrophages}

To test if gelatin nanospheres can act as carriers for local delivery of antibiotics, THP-1-derived macrophages were cultured in medium containing vancomycin-loaded gelatin nanospheres. The cellular uptake of vancomycin-loaded gelatin nanospheres was studied using fluorescence microscopy. In brief, $100 \mu \mathrm{g}$ of fluorescently labeled vancomycin was dissolved in $1 \mathrm{~mL}$ of a freshly prepared suspension of fluorescently labeled gelatin nanospheres in Milli-Q water $(18 \mathrm{mg} / \mathrm{mL})$ to form a suspension of vancomycin-loaded gelatin nanospheres. This suspension was equilibrated at $4^{\circ} \mathrm{C}$ for 12 hours to allow for adsorption of vancomycin to the gelatin nanospheres by polyion formation between vancomycin and gelatin macromers. Previously we showed that the affinity between vancomycin and gelatin nanospheres was high, resulting in the absence of any burst-type release of vancomycin. ${ }^{27,28}$ Therefore, we assume that the loading efficiency of the gelatin nanospheres was high and the amount of unbound vancomycin low. Vancomycin was added to the suspension of gelatin nanospheres in order to ensure that all vancomycin was confined within the colloidal suspension. THP-1 human monocytic cells were cultured in $500 \mu \mathrm{L}$ of culture medium, and differentiation into macrophages was induced with PMA. Subsequently, $5 \mu \mathrm{L}$ of the suspension of vancomycin-loaded gelatin nanospheres were added to the culture medium and the macrophages were cultured for 24 hours. THP-1-derived macrophages were also incubated with $5 \mu \mathrm{L}$ of vancomycin solution in Milli-Q water $(100 \mu \mathrm{g} / \mathrm{mL})$ for 24 hours as control experiment. After incubation for 24 hours, the cells were washed with PBS for 3 times and fixed with 4\% paraformaldehyde. The nucleic acid and actin skeleton of the cells was stained with DAPI and Alexa Fluor ${ }^{\circledR} 568$ Phalloidin (ThermoFisher Scientific), respectively. ProLong ${ }^{\circledR}$ Diamond Antifade Mountant was used to mount the cells for imaging. A series of Z-stack images were recorded and subsequently converted to $3 \mathrm{D}$ videos using the Leica LAS X software.

\section{Visualization of internalization of vancomycin-loaded gelatin nanospheres by macrophages in zebrafish larvae}

To study internalization of vancomycin-loaded gelatin nanospheres in vivo, we injected the suspension of vancomycinloaded gelatin nanospheres intramuscularly into the tail muscle tissue of zebrafish larvae of the transgenic line 
(fms:Gal4/UAS:mCherry) which express mCherry fluorescent protein in their macrophages, following the procedure described earlier. A solution of free vancomycin without gelatin nanospheres in Milli-Q water was also injected intramuscularly into a control group of larvae. The interaction between zebrafish macrophages and vancomycin-loaded gelatin nanospheres or free vancomycin was monitored using confocal microscopy at 24 hours post-injection as described above. A series of Z-stack images were recorded and subsequently converted to $3 \mathrm{D}$ videos using the Leica LAS X software.

\section{Visualization of co-localization of macrophages and bacteria in zebrafish larvae}

We used fluorescence microscopy (Leica LM80) to record the co-localization of macrophages and bacteria in 1-dayold zebrafish larvae. The zebrafish transgenic line (mpeg 1: Gal4/UAS: Kaede) and S. aureus RN4220 expressing $\mathrm{mCherry}$ fluorescent protein were used. The bacteria were cultured in tryptic soy broth for $\sim 3-4$ hours to reach the logarithmic growth phase at $37^{\circ} \mathrm{C}$. Subsequently, bacteria were collected by centrifugation, washed repeatedly with PBS, and resuspended in Milli-Q water to a concentration adjusted to $\sim 1,000$ colony-forming unit (CFU)/nL. Zebrafish larvae were dechorionized, anaesthetized with tricaine, and aligned in an agar plate containing E3 medium for injections. At 30 hours post-fertilization, $3 \mathrm{~nL}$ of the suspension of $S$. aureus bacteria was injected into the blood circulation of zebrafish larvae via the blood island ${ }^{46}$ to reach a challenge dose of $\sim 3,000$ CFU per larva, which was verified by quantitative culture of 6 randomly selected infected larvae crushed using a MagNA lyser (Roche, Almere, the Netherlands). At 32 hours post-fertilization, S. aureusinfected zebrafish larvae were imaged using fluorescence microscopy (Leica LM80, Eindhoven, the Netherlands) under bright field, and with the mCherry and the GFP filters under optimized setting.

\section{Treatment of S. aureus-infected zebrafish larvae with vancomycin using gelatin nanospheres as carriers}

To investigate if the antibacterial efficacy of vancomycin can be enhanced upon local delivery from vancomycinloaded gelatin nanospheres, we injected $\sim 3,000 \mathrm{CFU}$ of $S$. aureus ATCC 49230 expressing green fluorescent protein $^{49}$ intravenously into wild-type TL zebrafish larvae as described earlier, at 30 hours post-fertilization. After injection of the bacteria, the larvae were randomly divided into experimental groups for subsequent treatment of the infection. At 32 hours post-fertilization, so at 2 hours after infection, $3 \mathrm{~nL}$ of a freshly prepared suspension of fluorescently labeled gelatin nanospheres $(18 \mathrm{mg} / \mathrm{mL}$, in Milli-Q water) containing $0.13 \mathrm{mg} / \mathrm{mL}$ vancomycin, or $3 \mathrm{~nL}$ of $0.13 \mathrm{mg} / \mathrm{mL}$ vancomycin dissolved in Milli-Q water, or $3 \mathrm{~nL}$ of a freshly prepared suspension of gelatin nanospheres in Milli-Q, or $3 \mathrm{~nL}$ of Milli-Q water as mock treatment control, was injected into the blood circulation of infected larvae via the blood island. To prepare vancomycin-loaded gelatin nanospheres, vancomycin was dissolved in the liquid phase of the suspension of gelatin nanospheres. This vancomycinloaded gelatin nanosphere suspension was equilibrated at $4^{\circ} \mathrm{C}$ for 12 hours to allow for adsorption of vancomycin to the gelatin nanospheres through polyion formation between vancomycin and gelatin macromers. This vancomycin dose of $\sim 0.4 \mu \mathrm{g}$ per larva was selected since this dose failed to protect larvae against the aforementioned dose of $S$. aureus, based on our pilot experiments. An infection-free control group received an injection of Milli-Q water instead of S. aureus, and another injection of Milli-Q water instead of antibiotic treatment, at the respective time points. This control group was included to assess the possible harmful effect caused by the double injection procedure. Survival of the larvae in each group was monitored daily (heartbeat and movement) from the day of injection until 4 days post-injection.

\section{Statistics}

All statistics were carried out using Graphpad Prism version 7.0. Percent survival of zebrafish larvae was analyzed using the Kaplan-Meier method. Differences in survival between pairs of groups over the entire time period of the experiment were statistically analyzed using the Log-rank test method.

\section{Results \\ Characterization of fluorescently labeled gelatin nanospheres}

Fluorescent labeling of gelatin nanospheres with the far-red dye (DyLight ${ }^{\mathrm{TM}}$ 650) did not influence the morphology of the dehydrated nanospheres. The non-labeled and labeled nanospheres in dry state had a comparable size range (Figure 1). For gelatin nanospheres in swollen state (PBS buffer), the fluorescent labeling caused a slight increase in the average size of gelatin nanospheres from 329 to $387 \mathrm{~nm}$ (Table 1). The average size of the fluorescently labeled gelatin nanospheres 

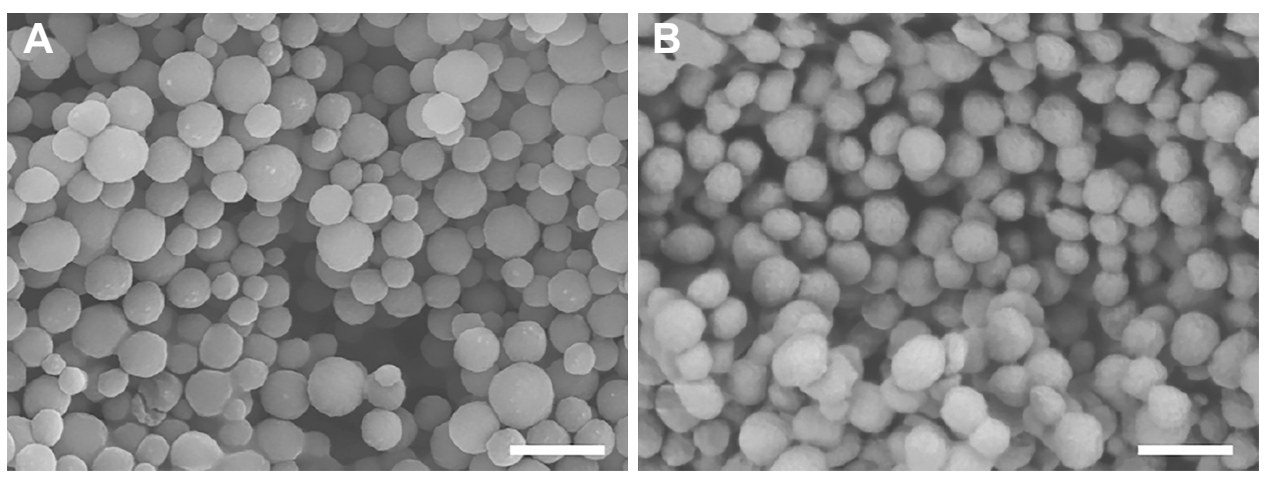

Figure I Scanning electronic micrograph of dehydrated gelatin nanospheres without (A) and with (B) fluorescent labeling. Scale bars represent $500 \mathrm{~nm}$.

in swollen state in Milli-Q water was $676 \mathrm{~nm}$. Both the size and charge of the gelatin nanospheres were smaller in PBS buffer with high ionic strength as compared to Milli-Q water, which has lower ionic strength. These effects were caused by screening of the negative charges along the gelatin macromers, thereby reducing the repulsive interactions between gelatin macromers.

\section{Distribution of gelatin nanospheres and their interactions with macrophages after intravenous injection into zebrafish larvae}

To investigate the distribution of gelatin nanospheres in the blood circulation of live animals and the elicited response of macrophages in vivo using confocal fluorescence microscopy, fluorescently labeled gelatin nanospheres were injected intravenously into the Duct of Cuvier of 3-day-old zebrafish larvae expressing Kaede fluorescent proteins (green) in their macrophages (Figure 2A and B; Figure $3 \mathrm{~A}$ and B). At 3 hours post-injection, the injected gelatin nanospheres had already spread throughout the blood vessels of the larvae (Figure 2C). The majority of the observed macrophages was patrolling the blood vessels, while few macrophages co-localized with gelatin nanospheres in the caudal blood vessel (Figure 2D, blue dashed box; Figure 2E; Video S1). However, the gelatin nanospheres that were not associated with macrophages

Table I Parameters of non-labeled and fluorescently labeled gelatin nanospheres in swollen state in different solutions

\begin{tabular}{|c|c|c|c|}
\hline \multirow[t]{2}{*}{ Parameters } & \multirow{2}{*}{$\begin{array}{l}\text { Non-labeled } \\
\text { PBS (I } 54 \mathrm{mM} \text {, } \\
\text { pH 7.4) }\end{array}$} & \multirow{2}{*}{$\begin{array}{l}\text { Fluorescent- } \\
\text { labeled } \\
\text { PBS }\end{array}$} & \multirow{2}{*}{$\begin{array}{l}\text { Fluorescent- } \\
\text { labeled } \\
\text { Milli-Q water } \\
\text { (pH 7) }\end{array}$} \\
\hline & & & \\
\hline Particle size $(\mathrm{nm})$ & $329 \pm 5$ & $387 \pm 7$ & $676 \pm 13$ \\
\hline$\zeta$-potential $(\mathrm{mV})$ & $-6.63 \pm 0.86$ & $-6.55 \pm 0.20$ & $-25.60 \pm 0.45$ \\
\hline
\end{tabular}

Abbreviation: PBS, phosphate buffered saline. seemed to be clustered, suggesting internalization by other non-labeled cell types such as neutrophils, as for instance also observed by other in vivo studies on the cellular uptake of silica or PLGA nanoparticles using zebrafish larvae. ${ }^{34,35}$ The agglomeration of the non-internalized gelatin nanospheres in the blood circulation may be caused by the high ionic strength of the blood. The high ionic strength of blood likely screened the repulsive interactions between the negatively charged gelatin nanospheres, ${ }^{22}$ as the zeta-potential of gelatin nanospheres in PBS buffer (with a similar ionic strength level compared to that of blood) was lower than that of gelatin nanospheres in Milli-Q water (Table 1). At 24 hours postinjection, the co-localization of the gelatin nanospheres and the macrophages was mainly observed in the area surrounding the yolk sac of the larvae (Figure 3C and D, blue dashed box; Figure 3E; Video S2).

\section{Distribution of gelatin nanospheres and their interactions with macrophages after intramuscular injection into zebrafish larvae}

In order to investigate the distribution of gelatin nanospheres in muscle tissue of the zebrafish larvae and the provoked response of macrophages in vivo using confocal fluorescence microscopy, fluorescently labeled gelatin nanospheres were injected intramuscularly into 3-day-old zebrafish larvae with macrophages expressing the Kaede green fluorescent protein (Figure 4B and C; Figure 5A and B). In contrast to intravenous injection of gelatin nanospheres which resulted in spreading of the gelatin nanospheres throughout the circulation (Figure 2C), the intramuscularly injected gelatin nanospheres only spread throughout the muscle tissue close to the injection site at 3 hours post-injection (Figure $4 \mathrm{~A}$ and D). Although some macrophages had already been attracted to this tissue area at this early time point, only few macrophages 

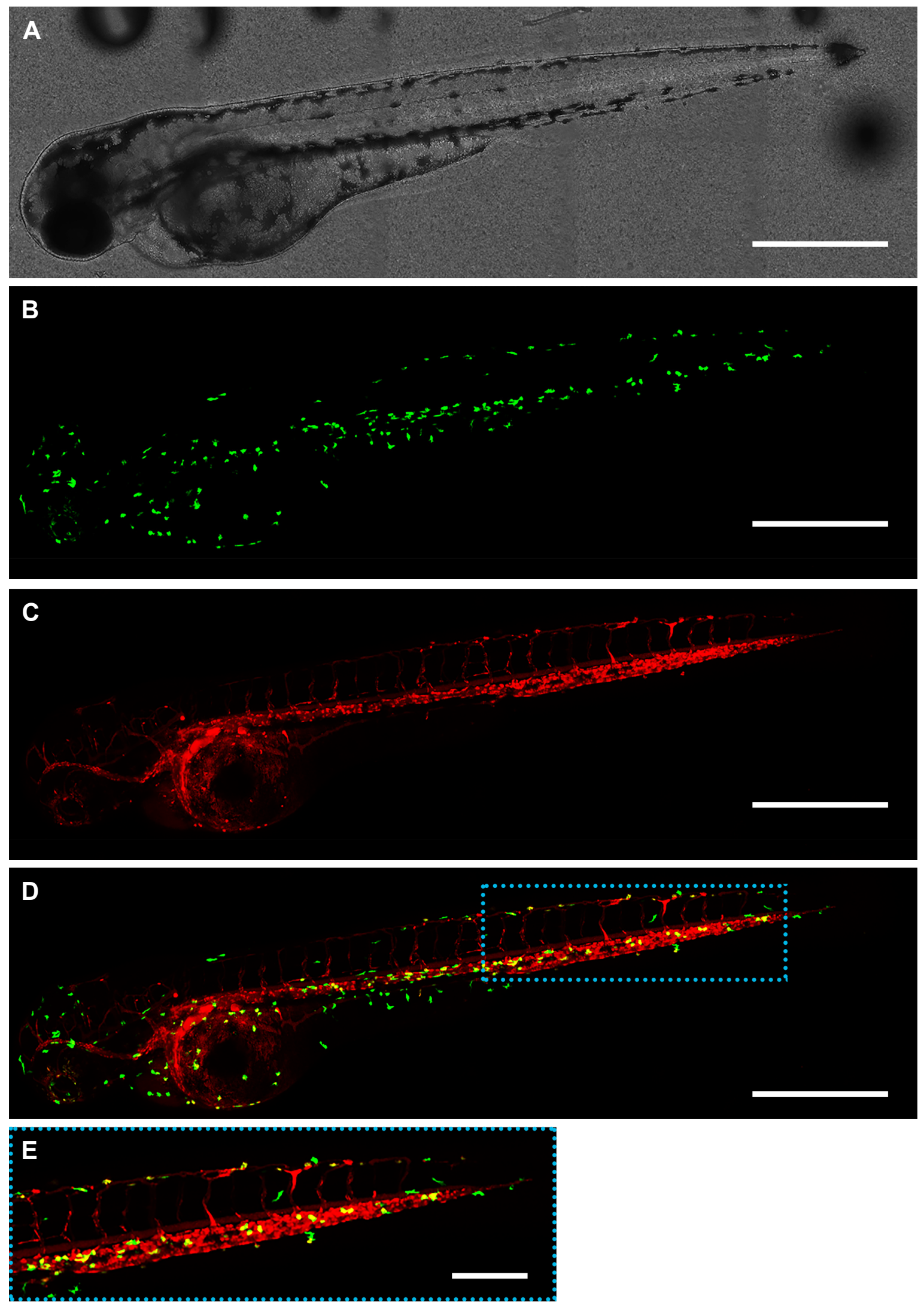

Figure 2 Distribution of zebrafish macrophages and gelatin nanospheres at 3 hours after intravenous injection of gelatin nanospheres into a 3-day-old zebrafish larva. (A) Bright field image; (B) green fluorescent macrophages; (C) red fluorescent gelatin nanospheres; (D) merged image of (B) and (C) with co-localization of gelatin nanospheres and macrophages depicted in yellow. Several macrophages co-localized with gelatin nanospheres in the caudal blood vessel of the larva (blue dashed box in $\mathbf{D}$ ). Scale bars represent $500 \mu \mathrm{m}$. (E) Magnification of the boxed area in (D). Scale bar represents $200 \mu \mathrm{m}$. 

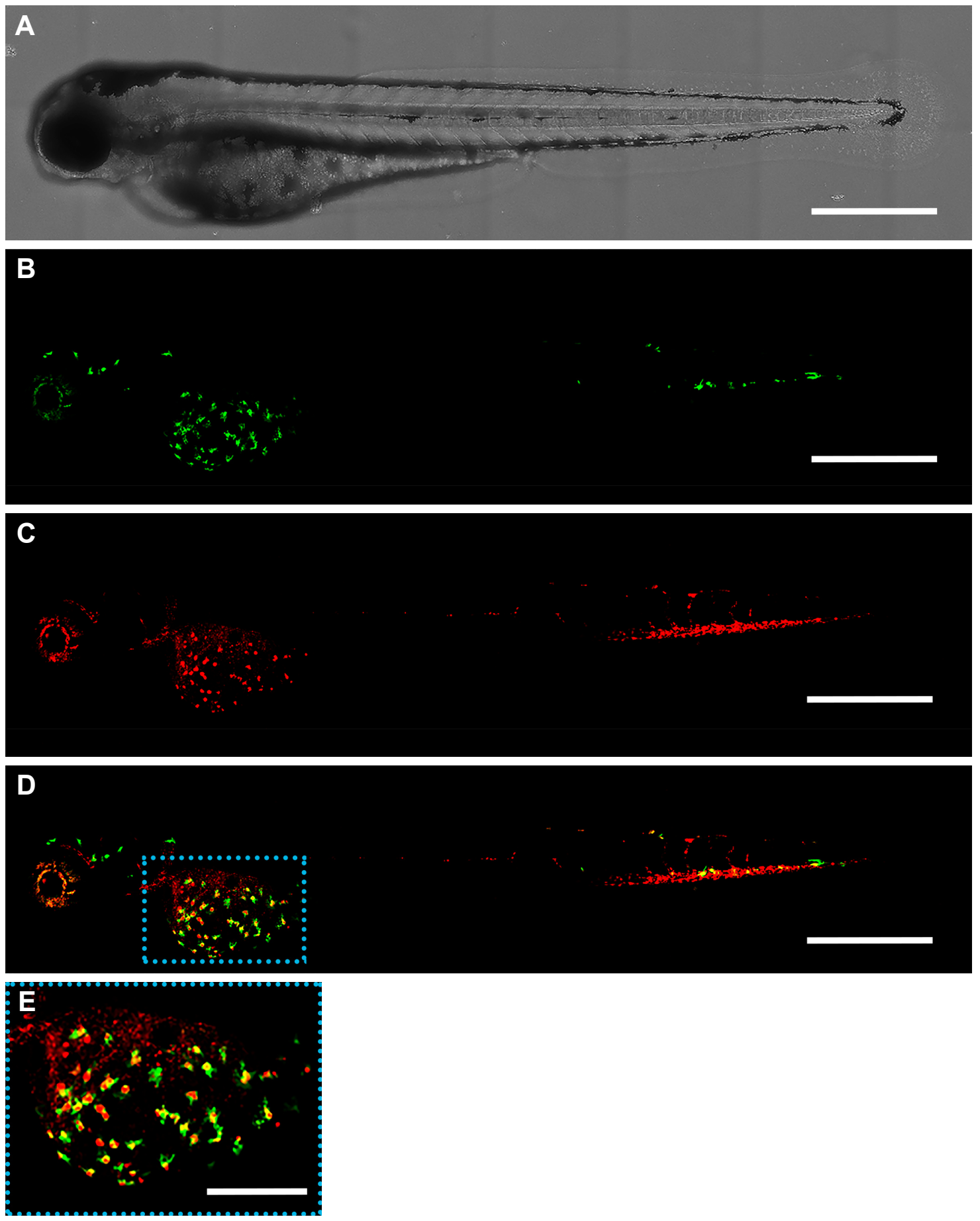

Figure 3 Distribution of zebrafish macrophages and gelatin nanospheres at 24 hours after intravenous injection of gelatin nanospheres into a 3-day-old zebrafish larva. (A) Bright field image; (B) green fluorescent macrophages; (C) red fluorescent gelatin nanospheres; (D) merged image of (B) and (C) with co-localization of gelatin nanospheres and macrophages depicted in yellow. The majority of the observed macrophages co-localized with gelatin nanospheres in the area surrounding the yolk sac of the larva (blue dashed box in D). Scale bars represent $500 \mu \mathrm{m}$. (E) Magnification of the boxed area in (D). Scale bar represents $200 \mu \mathrm{m}$.

seemed to co-localize with gelatin nanospheres (Figure 4E, white dashed box; Video S3).

At 24 hours post-injection, the original mass of gelatin nanospheres had been dispersed into separate clusters, but the majority of the gelatin nanospheres was still confined to the muscle tissue near the injection site and engulfed by macrophages (Figure 5C and D). The corresponding video (Video S4) confirmed that the gelatin nanospheres that co-localized with the zebrafish macrophages were internalized. 

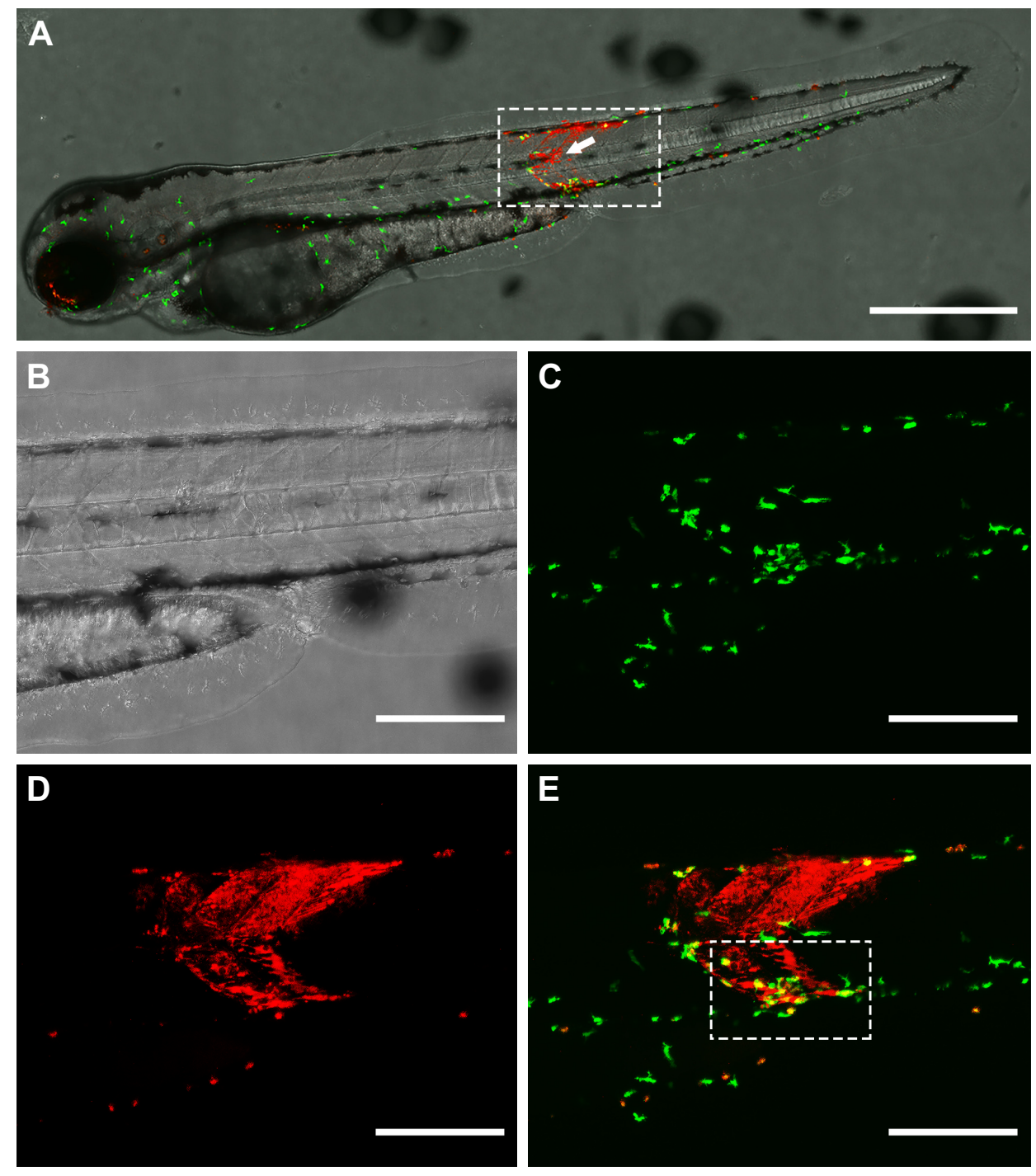

Figure 4 Distribution of zebrafish macrophages and gelatin nanospheres in the muscle tissue surrounding the injection site at 3 hours after intramuscular injection of gelatin nanospheres into a 3-day-old zebrafish larva. (A) Merged overview image with white dashed box indicating the tissue area of injection (injection site indicated by arrow). (B-E) Magnification of the boxed area of (A); (B) depicting the bright field image; (C) green fluorescent macrophages; (D) red fluorescent gelatin nanospheres; (E) merged image of $(\mathbf{C})$ and $(\mathbf{D})$ with co-localization of gelatin nanospheres and macrophages depicted in yellow. Several macrophages co-localized with gelatin nanospheres (E, white dashed box). Scale bar in (A) represents $500 \mu \mathrm{m}$ and in (B-E) $200 \mu \mathrm{m}$.

\section{Internalization of gelatin nanospheres and vancomycin-loaded gelatin nanospheres by THP-I-derived macrophages in vitro}

To assess whether internalization of gelatin nanospheres by macrophages as observed in the zebrafish larvae also would occur in human macrophages in vitro, we cultured THP1-derived macrophages in cell culture medium containing gelatin nanospheres. Internalization of gelatin nanospheres by these macrophages was indeed observed after 24 hours of culture (Figure 6A), which was confirmed by the corresponding video (Video S5).

To study the potential internalization of gelatin nanospheres by macrophages in vitro, THP-1-derived macrophages were cultured in medium containing vancomycin-loaded gelatin nanospheres. The vancomycin-loaded gelatin nanospheres were internalized by the macrophages after 24 hours of culture (Figure 6B; Video S6). The majority of the internalized vancomycin co-localized with the gelatin nanospheres intracellularly within the THP-1-derived macrophages, although fluorescent signal of vancomycin not associated with gelatin nanospheres was also detected in the cytosol of the macrophages. This suggests that this vancomycin was partially released intracellularly from gelatin nanospheres in the cytosol of the macrophages (Figure 6B). In the control group of macrophages cultured with free vancomycin without gelatin nanospheres as carriers, no internalized 

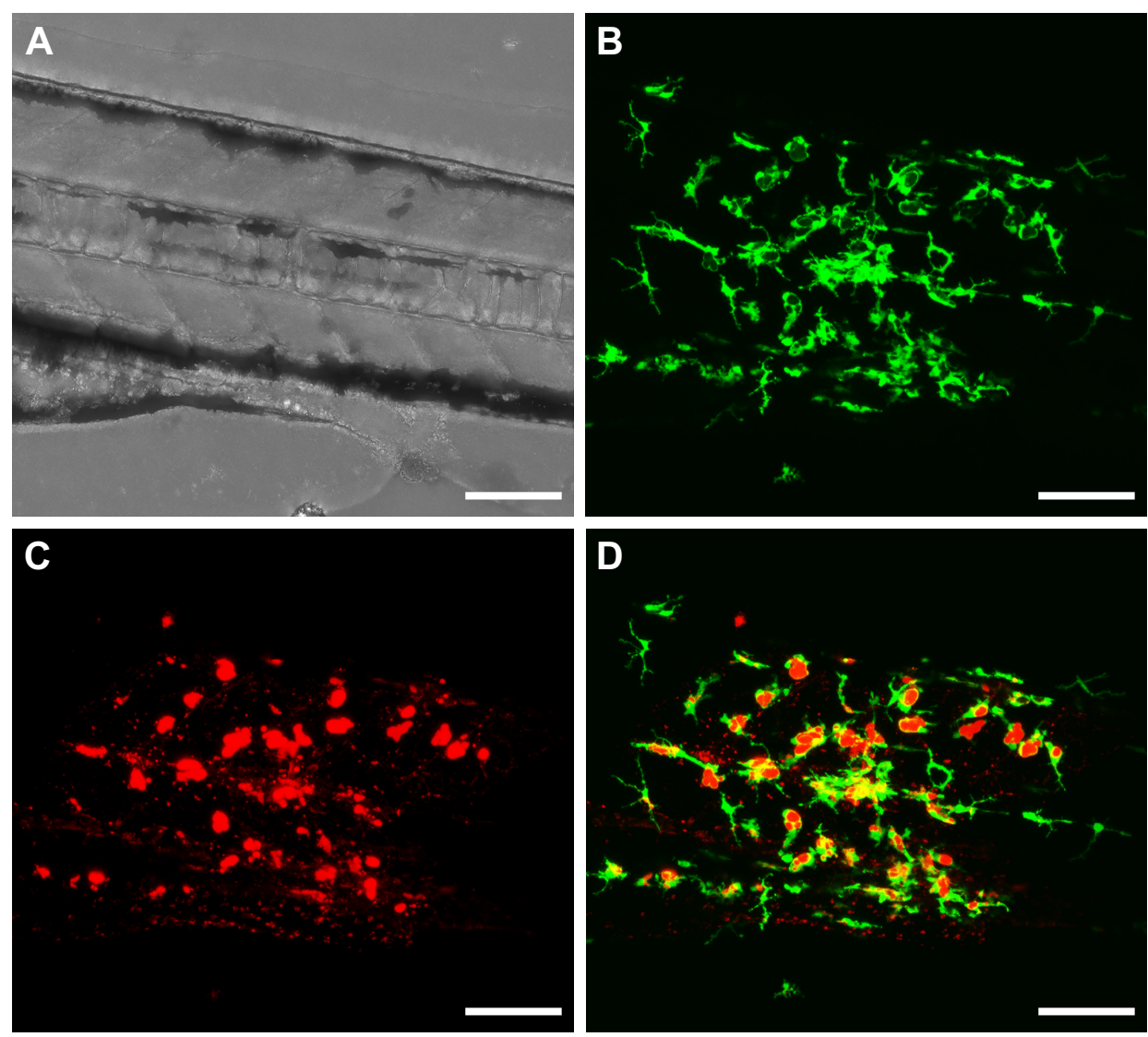

Figure 5 Co-localization of zebrafish macrophages and gelatin nanospheres in the muscle tissue surrounding the injection site at 24 hours after intramuscular injection of gelatin nanospheres into a 3-day-old zebrafish larva. (A) Bright field image; (B) green fluorescent macrophages; (C) red fluorescent gelatin nanospheres; (D) merged image of $(\mathbf{B})$ and $(\mathbf{C})$, with co-localization of macrophages and gelatin nanospheres depicted in yellow. The majority of the injected gelatin nanospheres was confined to the tissue area of injection and engulfed by macrophages, as shown in the merged image (D). Scale bars represent $100 \mu \mathrm{m}$.

vancomycin was detected inside the cells after 24 hours of culture (Figure 6C).

\section{Internalization of vancomycin-loaded gelatin nanospheres by macrophages of zebrafish larvae}

To study the potential delivery of vancomycin by using gelatin nanospheres into macrophages in vivo, we injected vancomycin-loaded gelatin nanospheres into the muscle tissue of 3-day-old zebrafish larvae with macrophages expressing mCherry fluorescent protein (red) and studied the provoked response of macrophages. At 24 hours post-injection, co-localization of vancomycin-loaded gelatin nanospheres and macrophages of the zebrafish larvae was observed, suggesting cellular internalization of the vancomycinloaded gelatin nanospheres (Figure 7A, co-localization indicated by white arrows; Figure 7B; Figure S3). Internalization of vancomycin-loaded gelatin nanospheres was also shown in the corresponding videos, since internalized vancomycin and gelatin nanospheres were observed within vacuole-like structures inside the zebrafish macrophages
(Videos S7 and S8). Upon injection of free vancomycin without gelatin nanospheres as carriers, co-localization of vancomycin and zebrafish macrophages was hardly observed (data not shown).

\section{Phagocytosis of bacteria by macrophages of zebrafish larvae}

To investigate the effect of treatment with vancomycinloaded gelatin nanospheres on intracellular $S$. aureus, we first assessed if macrophages in 1-day-old zebrafish larvae were able to phagocytose $S$. aureus. To this end, we injected S. aureus expressing mCherry fluorescent protein (red) intravenously into 1-day-old zebrafish larvae with macrophages expressing Kaede fluorescent protein (green) (Figure 8A). At 2 hours post-injection of bacteria, the majority of the bacteria co-localized with zebrafish macrophages as observed using fluorescence microscopy, suggesting that the bacteria were phagocytosed by the macrophages (Figure 8B-D). The bacteria that did not associate with macrophages also seemed to be clustered (Figure 8C), suggesting that they may have been phagocytosed by other phagocytes such as neutrophils, 

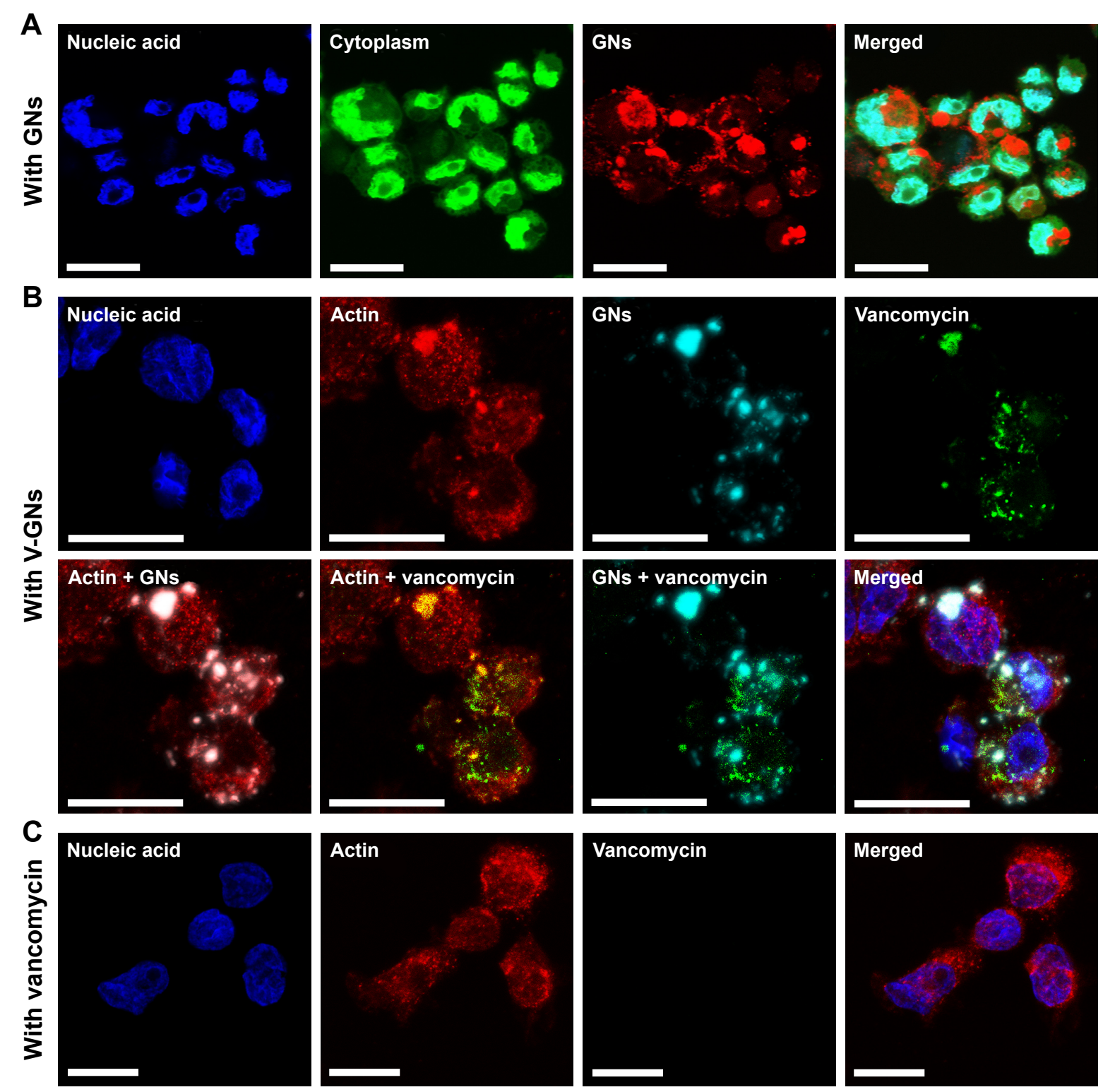

Figure 6 In vitro internalization of fluorescently labeled gelatin nanospheres (GNs, A) and GNs loaded with fluorescent-labeled vancomycin (V-GNs, B) by THP-I-derived macrophages after 24 hours of culture. Cells incubated with free vancomycin without GNs as carriers were used as controls (C). Nucleic acid of the cells was stained with DAPI/blue, and cytoplasm and actin skeleton with Cell-Tracker ${ }^{\mathrm{TM}}$ CMFDA/green (in A) and Alexa Fluor ${ }^{\circledR} 568$ Phalloidin/red (in B and C), respectively. Cell-Tracker ${ }^{\mathrm{TM}}$ CMFDA/green also stained nucleic acid of the cells. The fluorescent-labeled GNs were displayed in red (in $\mathbf{A}$ ) and in cyan (in B). Scale bars represent $20 \mu \mathrm{m}$ (in $\mathbf{A}$ and $\mathbf{B}$ ) and $10 \mu \mathrm{m}$ (in C).

which were non-labeled in the fish line used in this experiment). This early phagocytosis is in accordance with results reported in a study that used a similar zebrafish larvae $S$. aureus infection model. ${ }^{33}$ Thus, our model allowed studies on the therapeutic efficacy of vancomycin-loaded gelatin nanospheres against intracellular bacterial infection.

\section{Enhanced protection of zebrafish} larvae against $S$. aureus infection using vancomycin-loaded gelatin nanospheres

To assess the potential of gelatin nanospheres as carriers to enhance the antibacterial activity of vancomycin in vivo, we injected $S$. aureus intravenously into zebrafish larvae at 1 day post-fertilization and subsequently treated the infected larvae with a single intravenous injection of vancomycinloaded gelatin nanospheres, free vancomycin, or only gelatin nanospheres at 2 hours after the $S$. aureus injection. A dose of vancomycin of $0.4 \mathrm{ng}$ per larva was selected since this dose failed to protect the $S$. aureus-infected larvae when injected without any carrier and could thus be used to study the potential of gelatin nanospheres as carriers to improve the efficacy of vancomycin.

The dual injection procedure itself caused $20 \%$ of larvae in the infection-free control group to die at 1 day 

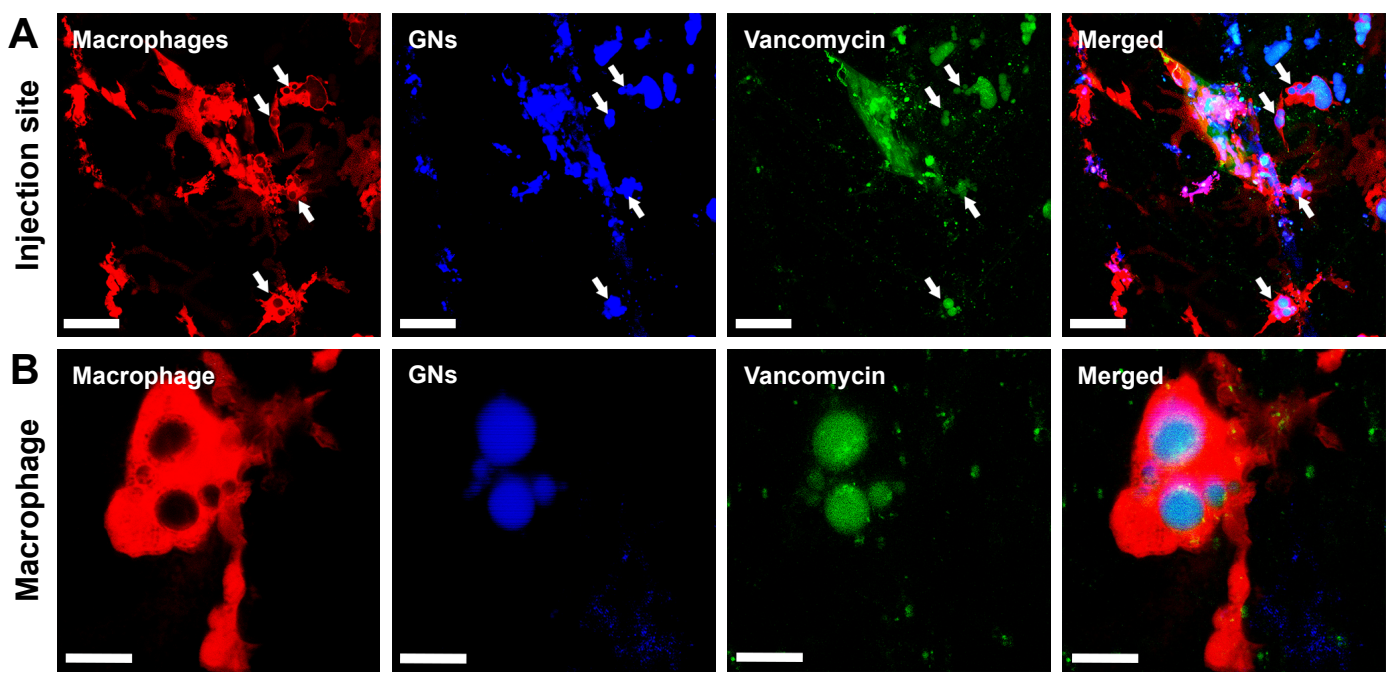

Figure 7 In vivo cellular internalization of fluorescently labeled gelatin nanospheres (GNs, blue) and fluorescent-labeled vancomycin (green) by fluorescent zebrafish macrophages (red) at 24 hours after intramuscular injection of vancomycin-loaded GNs into a I-day-old zebrafish larva. (A) Interactions between zebrafish macrophages and vancomycin-loaded GNs observed in the muscle tissue surrounding the injection site. The white arrows point at vacuole-like structures of macrophages containing internalized gelatin nanospheres and vancomycin. (B) High magnification of a zebrafish macrophage containing the internalized gelatin nanospheres and vancomycin. Scale bars in $\mathbf{A}$ and $\mathbf{B}$ represent 50 and $10 \mu \mathrm{m}$, respectively.
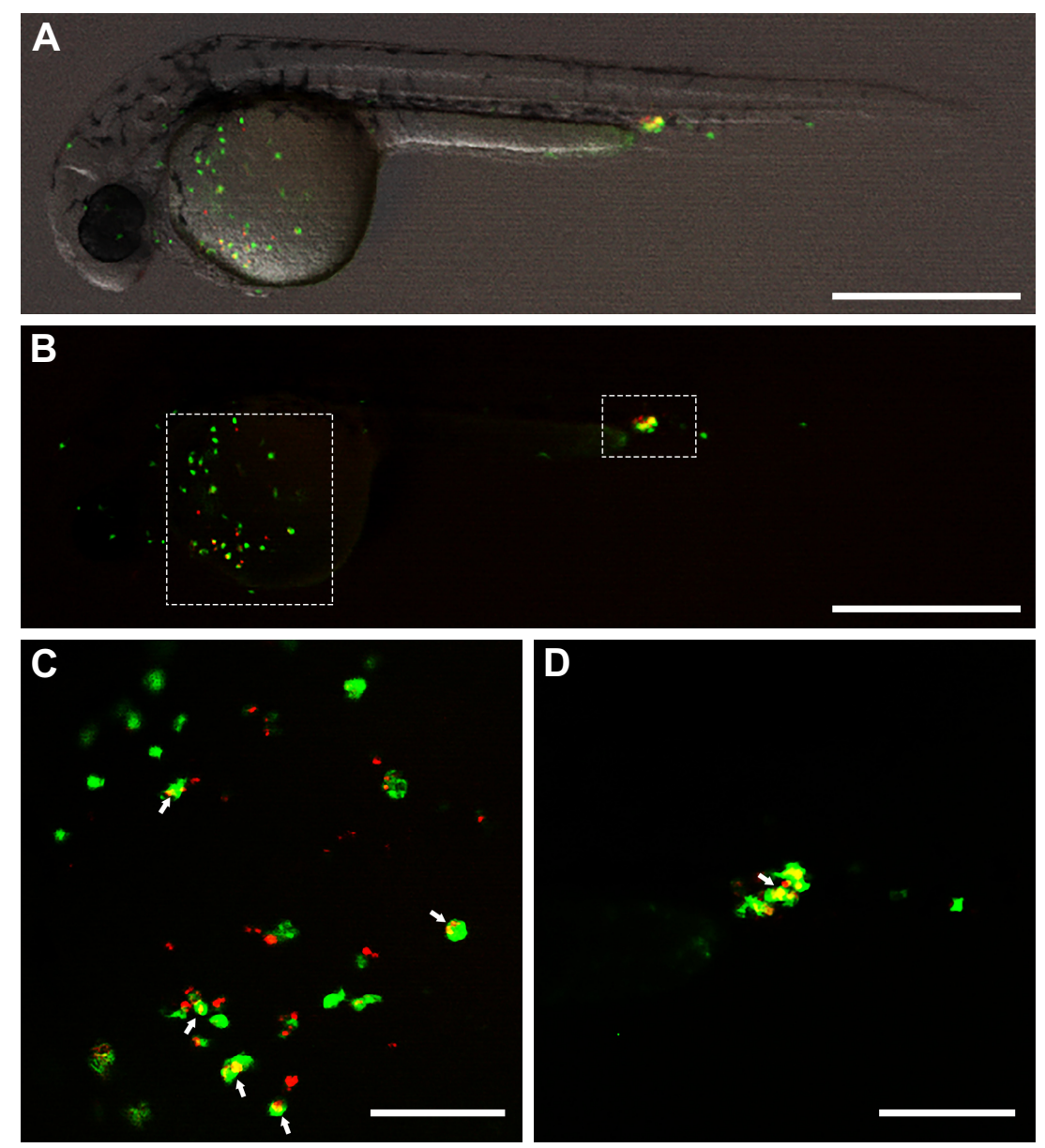

Figure 8 Co-localization of green fluorescent zebrafish macrophages (green) and red fluorescent S. aureus at 2 hours after intravenous injection of bacteria into a I-day-old zebrafish larva. The median dose of S. aureus assessed directly after injection in 6 control larvae was 3,000 CFU/larva. (A) Merged overview image; (B) distribution of the fluorescent macrophages and injected S. aureus; white dashed boxes indicate the areas of the recorded co-localization enlarged in $\mathbf{C}$ and $\mathbf{D}$; (C and $\mathbf{D})$ high magnification of the recorded co-localization, indicated by arrows. Scale bars in A-B and C-D represent 500 and $100 \mu \mathrm{m}$, respectively.

Abbreviation: $\mathrm{CFU}$, colony-forming unit. 
post-injection. Subsequently, survival in this infection-free group remained $80 \%$ until 4 days post-injection (Figure 9). Survival of the infected larvae receiving free vancomycin or only gelatin nanospheres dramatically decreased to $\sim 20 \%$ already at 1 day post-injection and decreased further during the following 3 days (Figure 9). Survival in these groups was not statistically different from that in the control group treated with Milli-Q water only ( $P=0.43$ and 0.64$)$. In contrast, overtime survival of larvae receiving vancomycin-loaded gelatin nanospheres was statistically significantly higher than that of larvae receiving free vancomycin $(P=0.01)$ (Figure 9). During the first 3 days post-injection, survival rate of larvae receiving vancomycin-loaded gelatin nanospheres was higher than that of the other groups, which, however, gradually decreased to a level similar to that of the other groups at 4 days post-injection. These results showed that treatment with vancomycin-loaded gelatin nanospheres delayed the mortality of $S$. aureus-infected zebrafish larvae compared to systemic administration of free vancomycin, although this single dose of vancomycin-loaded gelatin nanospheres was not sufficient to protect the larvae until the end of the experiment.

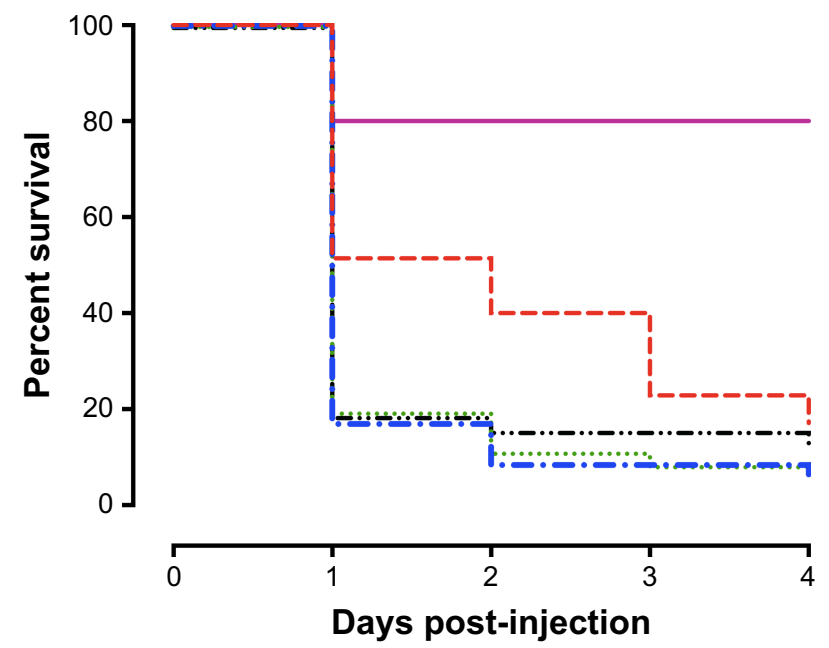

\begin{tabular}{|lll|}
\hline-- V-GNs & - Vancomycin
\end{tabular} ... GNs

Figure 9 Percent survival (\%) of S. aureus-infected zebrafish larvae receiving different treatments, monitored from the day of injection until 4 days post-injection. The median dose of $S$. aureus assessed directly after injection in control larvae was 3,200 CFU per larva. The infected larvae were treated with vancomycin-loaded gelatin nanospheres (V-GNs), free vancomycin, or only gelatin nanospheres (GNs). Injection of Milli-Q water was used as mock treatment. Larvae receiving two injections of Milli-Q water (infection-free) were used as controls for the injection procedure. The group size of infected larvae was between 32 and 35 larvae per group at the start of the experiment. The group size of infection-free larvae was 10 larvae. Survival of zebrafish larvae receiving locally delivered vancomycin (V-GNs) was statistically significantly higher than survival of zebrafish larvae receiving systemically delivered vancomycin $(P=0.0 \mathrm{I})$.

Abbreviation: CFU, colony-forming unit.

\section{Discussion}

The aim of the present study was to investigate the feasibility of gelatin nanospheres for local delivery of vancomycin in vivo. Using transgenic zebrafish larvae with macrophages expressing fluorescent proteins, we visualized in real time for the first time - the biodistribution of gelatin nanospheres in a live animal, which was dependent on the administration route (ie, intravenous or intramuscular injection). Moreover, our results showed that vancomycin-loaded gelatin nanospheres facilitated internalization of vancomycin by macrophages in zebrafish larvae in vivo and by THP-1-derived macrophages in vitro. Systemically delivered vancomycin, on the other hand, was hardly internalized by macrophages without the use of gelatin nanospheres. Treatment with a single dose of vancomycin-loaded gelatin nanospheres delayed the mortality of $S$. aureus-infected zebrafish larvae, indicating the improved therapeutic efficacy of vancomycin against (intracellular) $S$. aureus infection in vivo.

A major problem with respect to treatment of infection using antibiotics is the limited therapeutic efficacy of many antibiotics against pathogens inside cells. In the present study, we investigated if gelatin nanospheres can improve internalization of vancomycin by macrophages, since this cell type is known to host intracellularly surviving bacterial pathogens ${ }^{50}$ and plays an important role in the phagocytosis of nanocarriers used for local delivery. ${ }^{51,52}$ Our results showed in a qualitative manner that the use of gelatin nanospheres as carriers allowed for internalization of vancomycin by macrophages within zebrafish larvae in vivo and THP-1derived macrophages in vitro. These observations are in line with previously obtained data on the release kinetics of vancomycin from gelatin nanospheres, ${ }^{27,28}$ which indicated that vancomycin was adsorbed tightly to gelatin macromers in gelatin nanospheres. This strong affinity between gelatin nanospheres and vancomycin facilitated intracellular delivery of vancomycin following internalization of vancomycinloaded gelatin nanospheres by macrophages. Next, we studied whether the antibacterial efficacy of vancomycin was improved by the use of gelatin nanospheres as carriers using a zebrafish larvae $S$. aureus infection model. In this model, the majority of the infecting $S$. aureus had been phagocytosed prior to injection of antibiotics and nanospheres. Vancomycin was selected since this antibiotic is not effective in treating intracellular bacteria, ${ }^{7,9,41}$ which was confirmed by our study since a single dose of free vancomycin did not protect zebrafish larvae from $S$. aureus infection. Treatment with vancomycin-loaded gelatin nanospheres statistically significantly increased the survival over time 
of the $S$. aureus-infected larvae. In the first 3 days after treatment, higher survival levels were noted. The positive effect of vancomycin-loaded gelatin nanospheres on survival of zebrafish larvae can be attributed to either intracellular delivery and/or extracellular release of vancomycin from gelatin nanospheres contributing to the clearance of free bacteria in the blood circulation of larvae. Future studies should be executed in animal models, which allow quantification of intracellular versus extracellular delivery of vancomycin from gelatin nanospheres in order to elucidate the mechanism of action of vancomycin-loaded gelatin nanospheres for treatment of $S$. aureus infections.

The higher over-time survival of $S$. aureus-infected larvae treated with vancomycin-loaded gelatin nanospheres was characterized by high survival rates during the first 3 days after treatment. This seems to correlate with the relatively rapid release of vancomycin from gelatin nanospheres, as reported in our previous in vitro studies. ${ }^{27-29}$ The release kinetics of antibiotics from gelatin nanospheres depends strongly on the binding affinity of antibiotics to gelatin nanospheres. ${ }^{27}$ This affinity is controlled by several factors including the local $\mathrm{pH}$ and ionic strength as well as the enzymatic degradation of gelatin nanospheres. ${ }^{27}$ Monitoring the release kinetics of vancomycin from gelatin nanospheres in vivo was not possible for the current zebrafish larva model for various reasons. First, only small volumes of vancomycin $(3 \mathrm{~nL})$ can be administrated to the small larvae, which would result in final concentrations below the detection limit of common analytical techniques such as high-performance liquid chromatography. Moreover, quantification of internalized vancomycin using imaging analysis is not possible due to technical limitations. Hence, in vivo release kinetics should be studied using other animal models (eg, mouse) in order to optimize the formulation of vancomycin-loaded gelatin nanospheres.

Neutrophils and macrophages are suggested to be important niches of intracellular $S$. aureus in zebrafish larvae..$^{53}$ Thus, we speculate that low numbers of $S$. aureus may survive in neutrophils and macrophages of the larvae, forming reservoirs of infection at later time points. This is in accordance with the pathogenesis of $S$. aureus infection a similar larva model described previously. ${ }^{33}$ Therefore, repeated delivery of multiple doses of vancomycin-loaded gelatin nanospheres may improve the efficacy of vancomycin-loaded gelatin nanospheres on $S$. aureus infection in the zebrafish larva model. Phagolysosomal escape of bacteria into the cytosol of the niche cells is a crucial element in the pathogenesis of intracellular infection. This mechanism has been reported for S. aureus as well. ${ }^{54}$ Therefore, cytosolic release of antibiotics is desired to combat the bacteria residing in the cytosol of the cells. Interestingly, in the present study, a minor portion of the internalized vancomycin was observed separate from gelatin nanospheres within the cytosol of THP-1-derived macrophages in vitro, suggesting that gelatin nanosphere-based systems are suitable for cytosolic release of antibiotics. These data are, however, based on qualitative observation only and need quantitative confirmation in suitable larger animal models.

Several other intracellular delivery systems for antibiotics have been reported recently. For instance, a liposome-based system for intracellular delivery of vancomycin against $S$. aureus infection has been developed and successfully tested in a mouse model. ${ }^{41}$ However, liposomes have poor stability in biological fluids and may not be protective for the loaded drugs. ${ }^{12}$ Meanwhile, the drug payload of liposome-based systems is relatively low. ${ }^{11}$ Synthetic polymer nanoparticles are more stable than liposomes, but several types of polymeric nanoparticles are potentially toxic. ${ }^{12}$ The suitability of gelatin nanospheres as carriers for growth factors facilitating bone regeneration has been shown in various animal models such as rats, ${ }^{23,24}$ illustrating the biocompatibility of gelatin nanospheres and effectiveness of release of their bioactive cargos. The present study provides evidence that gelatin nanospheres can be used as nanocarriers to facilitate local delivery of antibacterial agents such as vancomycin. These nanospheres might also be used for local delivery of other types of antibiotics (eg, aminoglycosides or macrolides) or antimicrobial peptides. In that way, our gelatin nanosphere-based antibiotic delivery system may improve the efficacy of antibiotic delivery to eradicate intracellular staphylococci, possibly even antibiotic-tolerant bacterial phenotypes such as small colony variants of $S$. aureus ${ }^{55}$ or other intracellular pathogens such as Mycobacterium tuberculosis. ${ }^{56}$ As a consequence, the treatment of infections associated with intracellular bacteria may be improved considerably. Alternatively, gelatin nanospheres loaded with antibiotics can also be incorporated within coatings on medical implants and devices ${ }^{28}$ to tailor the release of antibiotics from implant surfaces to treat osteomyelitis and biomaterial-associated infections.

\section{Conclusion}

In the present study, we analyzed the biodistribution of gelatin nanospheres in zebrafish larvae in real time and showed that their distribution depended on the route of administration (ie, intravenous or intramuscular injection). The gelatin nanospheres were shown to be internalized by macrophages within zebrafish larvae and by THP-1-derived macrophages. Gelatin nanospheres facilitated internalization of vancomycin by macrophages both in vitro and in vivo, as opposed to poor internalization of systemically 
delivered vancomycin without gelatin nanospheres. Moreover, survival of zebrafish larvae infected with $S$. aureus was significantly higher following local delivery of vancomycin-loaded gelatin nanospheres as compared to survival after systemic delivery of free vancomycin over time, in particularly during the first 3 days. The present study demonstrates that gelatin nanospheres are promising nanocarriers to facilitate local delivery of vancomycin to treat infections.

\section{Acknowledgment}

The authors would like to thank Prof Dr Graham Lieschke from Monash University, Australia, and Prof Dr Steven Renshaw from Sheffield University, UK, for providing the zebrafish transgenic lines (mpeg1:Gal4/UAS:Kaede) and (fms:Gal4/UAS:mCherry), respectively.

\section{Disclosure}

Xiaolin Zhang was financially supported by IBIZA project of the BioMedical Material (BMM) program, co-funded by the Dutch Ministry of Economic Affairs. Jiankang Song was financially supported by the Chinese Scholarship Council under foundation number 201206150058 . The authors report no other conflicts of interest in this work.

\section{References}

1. Garzoni C, Kelley WL. Staphylococcus aureus: new evidence for intracellular persistence. Trends Microbiol. 2009;17(2):59-65.

2. Tong SY, Davis JS, Eichenberger E, Holland TL, Fowler VG Jr. Staphylococcus aureus infections: epidemiology, pathophysiology, clinical manifestations, and management. Clin Microbiol Rev. 2015;28(3): 603-661.

3. Moriarty TF, Kuehl R, Coenye T, et al. Orthopaedic device-related infection: current and future interventions for improved prevention and treatment. EFORT Open Rev. 2016;1(4):89-99.

4. Ellington JK, Harris M, Hudson MC, Vishin S, Webb LX, Sherertz R. Intracellular Staphylococcus aureus and antibiotic resistance: implications for treatment of staphylococcal osteomyelitis. J Orthop Res. 2006; 24(1):87-93.

5. Riool M, de Boer L, Jaspers V, et al. Staphylococcus epidermidis originating from titanium implants infects surrounding tissue and immune cells. Acta Biomater. 2014;10(12):5202-5212.

6. Boelens JJ, Dankert J, Murk JL, et al. Biomaterial-associated persistence of Streptococcus epidermidis in pericatheter macrophages. J Infect Dis. 2000;181(4):1337-1349.

7. Broekhuizen CAN, de Boer L, Schipper K, et al. Staphylococcus epidermidis is cleared from biomaterial implants but persists in peri-implant tissue in mice despite rifampicin/vancomycin treatment. J Biomed Mater Res A. 2008;85A(2):498-505.

8. Zaat SAJ, Broekhuizen CAN, Riool M. Host tissue as a niche for biomaterial-associated infection. Future Microbiol. 2010;5(8):1149-1151.

9. Barcia-Macay M, Seral C, Mingeot-Leclercq MP, Tulkens PM, Van Bambeke F. Pharmacodynamic evaluation of the intracellular activities of antibiotics against Staphylococcus aureus in a model of THP-1 macrophages. Antimicrob Agents Chemother. 2006;50(3):841-851.

10. Carryn S, Van Bambeke F, Mingeot-Leclereq MP, Tulkens PM. Comparative intracellular (THP-1 macrophage) and extracellular activities of beta-lactams, azithromycin, gentamicin, and fluoroquinolones against Listeria monocytogenes at clinically relevant concentrations. Antimicrob Agents Chemother. 2002;46(7):2095-2103.
11. Abed N, Couvreur P. Nanocarriers for antibiotics: a promising solution to treat intracellular bacterial infections. Int J Antimicrob Agents. 2014:43(6):485-496.

12. Xiong MH, Bao Y, Yang XZ, Zhu YH, Wang J. Delivery of antibiotics with polymeric particles. Adv Drug Delivery Rev. 2014;78:63-76.

13. Ruszczak Z, Friess W. Collagen as a carrier for on-site delivery of antibacterial drugs. Adv Drug Delivery Rev. 2003;55(12):1679-1698.

14. Ueng SW, Lee MS, Lin SS, Chan EC, Liu SJ. Development of a biodegradable alginate carrier system for antibiotics and bone cells. J Orthop Res. 2007;25(1):62-72.

15. Lecaroz MC, Blanco-Prieto MJ, Campanero MA, Salman H, Gamazo C. Poly(D,L-lactide-coglycolide) particles containing gentamicin: pharmacokinetics and pharmacodynamics in Brucella melitensis-infected mice. Antimicrob Agents Chemother. 2007;51(4):1185-1190.

16. Khalil IR, Burns AT, Radecka I, et al. Bacterial-derived polymer poly-y-glutamic acid (y-PGA)-based micro/nanoparticles as a delivery system for antimicrobials and other biomedical applications. Int $J \mathrm{Mol}$ Sci. 2017;18(2):pii:E313.

17. Liu H, Slamovich EB, Webster TJ. Less harmful acidic degradation of poly(lacticco-glycolic acid) bone tissue engineering scaffolds through titania nanoparticle addition. Int J Nanomedicine. 2006;1(4): $541-545$.

18. Santoro M, Tatara AM, Mikos AG. Gelatin carriers for drug and cell delivery in tissue engineering. $J$ Control Release. 2014;190:210-218.

19. Song J, Leeuwenburgh SC. Sustained delivery of biomolecules from gelatin carriers for applications in bone regeneration. Ther Deliv. 2014; 5(8):943-958.

20. Foox M, Zilberman M. Drug delivery from gelatin-based systems. Expert Opin Drug Deliv. 2015;12(9):1547-1563.

21. Su K, Wang C. Recent advances in the use of gelatin in biomedical research. Biotechnol Lett. 2015;37(11):2139-2145.

22. Wang H, Boerman OC, Sariibrahimoglu K, Li Y, Jansen JA, Leeuwenburgh SC. Comparison of micro- vs. nanostructured colloidal gelatin gels for sustained delivery of osteogenic proteins: bone morphogenetic protein-2 and alkaline phosphatase. Biomaterials. 2012;33(33):8695-8703.

23. van der Stok J, Wang H, Amin Yavari S, et al. Enhanced bone regeneration of cortical segmental bone defects using porous titanium scaffolds incorporated with colloidal gelatin gels for time- and dose-controlled delivery of dual growth factors. Tissue Eng Part A. 2013;19(23-24):2605-2614.

24. Wang H, Zou Q, Boerman OC, et al. Combined delivery of BMP-2 and bFGF from nanostructured colloidal gelatin gels and its effect on bone regeneration in vivo. $J$ Control Release. 2013;166(2):172-181.

25. Doi N, Jo JI, Tabata Y. Preparation of biodegradable gelatin nanospheres with a narrow size distribution for carrier of cellular internalization of plasmid DNA. J Biomater Sci Polym Ed. 2012;23(8):991-1004.

26. Ishikawa $\mathrm{H}$, Nakamura $\mathrm{Y}$, Jo J, Tabata Y. Gelatin nanospheres incorporating siRNA for controlled intracellular release. Biomaterials. 2012; 33(35):9097-9104.

27. Song JK, Odekerken JCE, Lowik DWPM, et al. Influence of the molecular weight and charge of antibiotics on their release kinetics from gelatin nanospheres. Macromol Biosci. 2015;15(7):901-911.

28. Song J, Chen Q, Zhang Y, et al. Electrophoretic deposition of chitosan coatings modified with gelatin nanospheres to tune the release of antibiotics. ACS Appl Mater Interfaces. 2016;8(22):13785-13792.

29. Song J, Klymov A, Shao J, et al. Electrospun nanofibrous silk fibroin membranes containing gelatin nanospheres for controlled delivery of biomolecules. Adv Healthc Mater. 2017;6(14):1-10.

30. Wang H, Hansen MB, Lowik DW, et al. Oppositely charged gelatin nanospheres as building blocks for injectable and biodegradable gels. Adv Mater. 2011;23(12):H119-H124.

31. Nahar M, Dubey V, Mishra D, Mishra PK, Dube A, Jain NK. In vitro evaluation of surface functionalized gelatin nanoparticles for macrophage targeting in the therapy of visceral leishmaniasis. $J$ Drug Target. 2010;18(2):93-105.

32. Veneman WJ, Marin-Juez R, de Sonneville J, et al. Establishment and optimization of a high throughput setup to study Staphylococcus epidermidis and Mycobacterium marinum infection as a model for drug discovery. J Vis Exp. 2014;(88):e51649. 
33. Prajsnar TK, Cunliffe VT, Foster SJ, Renshaw SA. A novel vertebrate model of Staphylococcus aureus infection reveals phagocyte-dependent resistance of zebrafish to non-host specialized pathogens. Cell Microbiol. 2008;10(11):2312-2325.

34. Fenaroli F, Westmoreland D, Benjaminsen J, et al. Nanoparticles as drug delivery system against tuberculosis in zebrafish embryos: direct visualization and treatment. ACS Nano. 2014;8(7):7014-7026.

35. Sharif F, Porta F, Meijer AH, Kros A, Richardson MK. Mesoporous silica nanoparticles as a compound delivery system in zebrafish embryos. Int J Nanomedicine. 2012;7:1875-1890.

36. Strahle U, Scholz S, Geisler R, et al. Zebrafish embryos as an alternative to animal experiments - a commentary on the definition of the onset of protected life stages in animal welfare regulations. Reprod Toxicol. 2012;33(2):128-132.

37. Renshaw SA, Trede NS. A model 450 million years in the making: zebrafish and vertebrate immunity. Dis Model Mech. 2012;5(1): $38-47$.

38. Goldsmith P. Zebrafish as a pharmacological tool: the how, why and when. Curr Opin Pharmacol. 2004;4(5):504-512.

39. Delvecchio C, Tiefenbach J, Krause HM. The zebrafish: a powerful platform for in vivo, HTS drug discovery. Assay Drug Dev Technol. 2011;9(4):354-361.

40. Zhang X, Stockhammer OW, de Boer L, et al. The zebrafish embryo as a model to quantify early inflammatory cell responses to biomaterials. J Biomed Mater Res A. 2017;105(9):2522-2532.

41. Surewaard BGJ, Deniset JF, Zemp FJ, et al. Identification and treatment of the Staphylococcus aureus reservoir in vivo. J Exp Med. 2016; 213(7):1141-1151.

42. Brand M, Granto M. Keeping and raising zebrafish. In: NüssleinVolhard C, Dahm R, editors. Zebrafish, A Practical Approach. Oxford: Oxford University Press; 2002:7-37.

43. Song J. Nanostructured carriers for the delivery of antibacterial agents. $\mathrm{PhD}$ thesis, Department of Biomaterials, Radboud University Nijmegen; 2016.

44. Gray C, Loynes CA, Whyte MKB, Crossman DC, Renshaw SA, Chico TJA. Simultaneous intravital imaging of macrophage and neutrophil behaviour during inflammation using a novel transgenic zebrafish. Thromb Haemost. 2011;105(5):811-819.
45. Ellett F, Pase L, Hayman JW, Andrianopoulos A, Lieschke GJ. mpeg1 promoter transgenes direct macrophage-lineage expression in zebrafish. Blood. 2011;117(4):E49-E56.

46. Benard EL, van der Sar AM, Ellett F, Lieschke GJ, Spaink HP, Meijer AH. Infection of zebrafish embryos with intracellular bacterial pathogens. $J$ Vis Exp. 2012;(61):e3781.

47. Ando R, Hama H, Yamamoto-Hino M, Mizuno H, Miyawaki A. An optical marker based on the UV-induced green-to-red photoconversion of a fluorescent protein. Proc Natl Acad Sci U S A. 2002;99(20): 12651-12656

48. Lombardo VA, Sporbert A, Abdelilah-Seyfried S. Cell tracking using photoconvertible proteins during zebrafish development. $J$ Vis Exp. 2012;(67):e4350.

49. Riool M, Dirks AJ, Jaspers V, et al. A chlorhexidine-releasing epoxybased coating on titanium implants prevents Staphylococcus aureus experimental biomaterial-associated infection. Eur Cell Mater. 2017;33: 143-157.

50. Gordon S. The macrophage: past, present and future. Eur J Immunol. 2007;37:S9-S17.

51. Oh N, Park JH. Endocytosis and exocytosis of nanoparticles in mammalian cells. Int J Nanomedicine. 2014;9:51-63.

52. Sahay G, Alakhova DY, Kabanov AV. Endocytosis of nanomedicines. J Control Release. 2010;145(3):182-195.

53. Prajsnar TK, Hamilton R, Garcia-Lara J, et al. A privileged intraphagocyte niche is responsible for disseminated infection of Staphylococcus aureus in a zebrafish model. Cell Microbiol. 2012;14(10):1600-1619.

54. Grosz M, Kolter J, Paprotka K, et al. Cytoplasmic replication of Staphylococcus aureus upon phagosomal escape triggered by phenol-soluble modulin alpha. Cell Microbiol. 2014;16(4):451-465.

55. Garcia LG, Lemaire S, Kahl BC, et al. Antibiotic activity against smallcolony variants of Staphylococcus aureus: review of in vitro, animal and clinical data. J Antimicrob Chemother. 2013;68(7):1455-1464.

56. Russell DG. Mycobacterium tuberculosis: here today, and here tomorrow. Nat Rev Mol Cell Biol. 2001;2(8):569-577. 


\section{Supplementary materials}

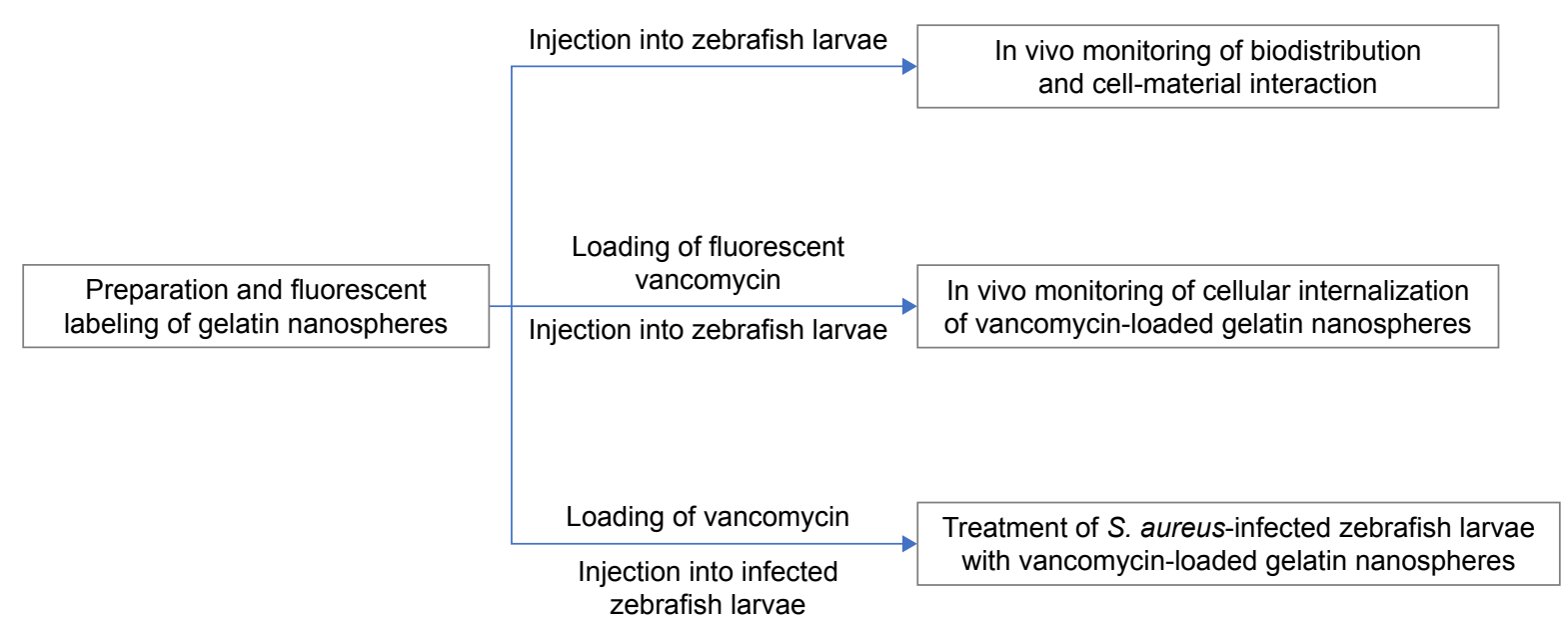

Figure SI The schematic experimental setup of the present study.
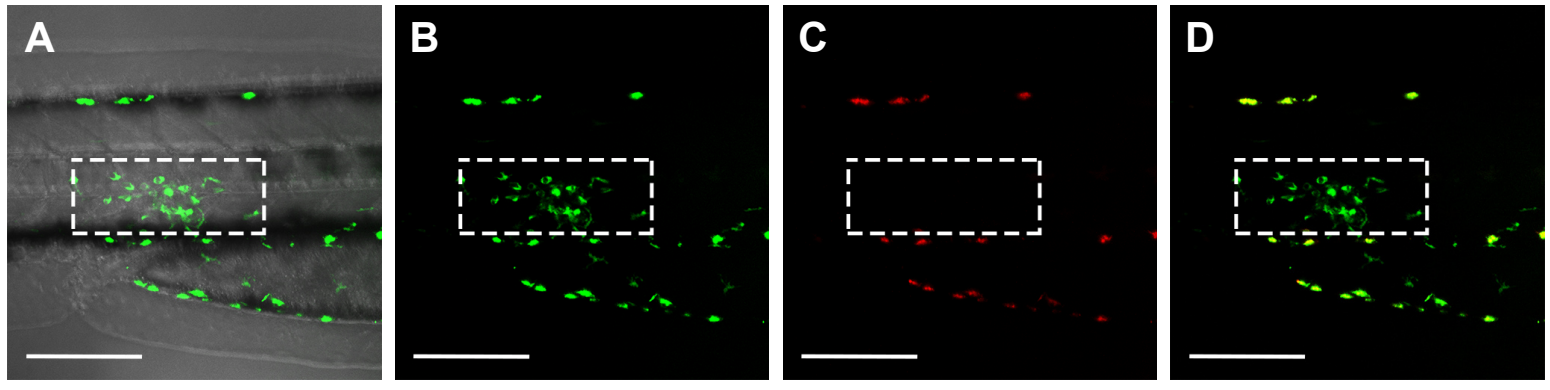

Figure S2 Detection of the Kaede fluorescent protein expressed in zebrafish macrophages at 3 hours post-intramuscular injection of PBS into a 3-day-old transgenic zebrafish larva (mpeg I: Kaede). The Kaede fluorescent protein may undergo an irreversible photoconversion from green to red fluorescence upon UV illumination (350-400 nm), depending on the exposure time and energy levels. (A) Merged image of the bright field and green channel image; (B) green channel image; (C) red channel image; (D) merged image of (B) and (C). The white dashed boxes indicate the location of the Kaede protein expressing macrophages in the muscle tissue area of injection. After illumination, no red signal was detected in these macrophages in the red channel (C), indicating that no color conversion of Kaede from green to red occurred under the setting used in the present study. Although the pigment cells of these zebrafish embryos show autofluorescence both in green and in red (fluorescence detected outside of the white dashed box in B-D), they mainly lined up at the border of the embryo trunk. They were not motile and not present in the area of injection. Therefore, the autofluorescence of pigment cells did not influence the recording of the macrophage responses in muscle tissue. Scale bars represent $100 \mu \mathrm{m}$.

Abbreviations: PBS, phosphate buffered saline; UV, ultra violet.
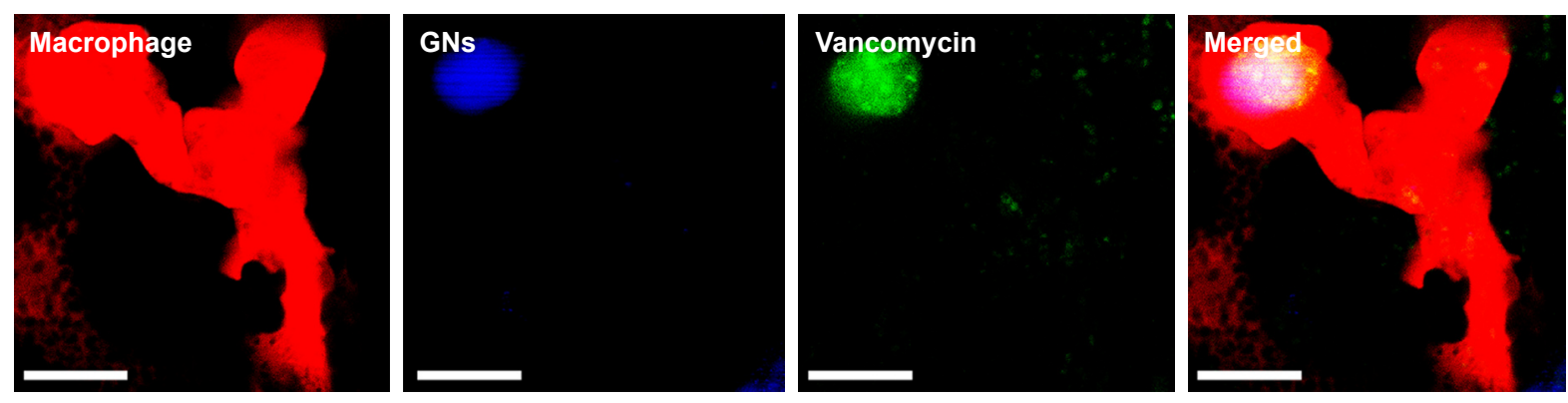

Figure S3 A zebrafish macrophage (red) containing internalized fluorescently labeled gelatin nanospheres (GNs, blue) and fluorescent-labeled vancomycin (green) at 24 hours after intramuscular injection of vancomycin-loaded GNs into a 3-day-old zebrafish larva. Scale bars represent $10 \mu \mathrm{m}$. 
Video SI Three-dimensional (3D) video showing the distribution of fluorescently labeled gelatin nanospheres (red) and Kaede fluorescent protein expressing macrophages (green) at 3 hours post-intravenous injection of gelatin nanospheres into a 3-day-old zebrafish larva. Parts of the embryo such as the yolk and head were not entirely shown in this video since the maximum depth of the employed Z-stack was limited. This 3D video was converted from a series of Z-stack images shown in Figure 2. Scale bar represents $500 \mu \mathrm{m}$.

Video S2 Three-dimensional (3D) video showing the distribution of fluorescently labeled gelatin nanospheres (red) and Kaede fluorescent protein expressing macrophages (green) at 24 hours post-intravenous injection of gelatin nanospheres into a 3-day-old zebrafish larva. Co-localization of gelatin nanospheres and macrophages was clearly observed in the area surrounding the yolk of the embryo. Parts of the embryo such as the yolk and head were not entirely shown in this video since the maximum depth of the employed $Z$-stack was limited. This 3D video was converted from a series of the Z-stack images shown in Figure 3. Scale bar represents $500 \mu \mathrm{m}$.

Video S3 Three-dimensional (3D) video showing the distribution of fluorescently labeled gelatin nanospheres (red) and Kaede fluorescent protein expressing zebrafish macrophages (green) in the muscle tissue of injection at 3 hours postintramuscular injection of gelatin nanospheres into a 3-day-old zebrafish larva. This video was converted from a series of the Z-stack images shown in Figure 4. Scale bar represents $200 \mu \mathrm{m}$

Video S4 Three-dimensional (3D) video showing the distribution of fluorescently labeled gelatin nanospheres (red) and Kaede fluorescent protein expressing zebrafish macrophages (green) in the muscle tissue of injection at 24 hours postintramuscular injection of gelatin nanospheres into a 3-day-old zebrafish larva. The majority of the injected gelatin nanospheres was engulfed by the macrophages. This video was converted from a series of the Z-stack images shown in Figure 5. Scale bar represents $100 \mu \mathrm{m}$.
Video S5 Three-dimensional (3D) video showing the internalization of fluorescently labeled gelatin nanospheres (red) by THP-I macrophages after 24 hours of culture. The cells appeared turquoise due to the merge of the colors of their nucleic acid which was stained both by DAPI and Cell-Tracker ${ }^{\mathrm{TM}}$ CMFDA/green. Their cytoplasm was stained by Cell-Tracker ${ }^{\mathrm{TM}}$ CMFDA/green. Scale bar represents $50 \mu \mathrm{m}$.

Video S6 Three-dimensional (3D) video showing the internalization of vancomycinloaded gelatin nanospheres by THP-I macrophages after 24 hours of culture. The nucleic acid and actin of the cells were stained by DAPI and Alexa Fluor ${ }^{\circledR}$ 594 Phalloidin, respectively. Fluorescent-labeled vancomycin and gelatin nanospheres were shown in green and cyan, respectively. This video was converted from a series of the Z-stack images shown in Figure 6. Scale bar represents $10 \mu \mathrm{m}$.

Video S7 Three-dimensional (3D) video showing the internalization of fluorescentlabeled vancomycin (green) in vacuole-like structures of zebrafish macrophage expressing mCherry fluorescent proteins (red) at 24 hours post-intramuscular injection of vancomycin-loaded gelatin nanospheres into a 3-day-old zebrafish larva. This video was converted from a series of the Z-stack images shown in (B) in Figure 7, excluding the blue channel images of fluorescently labeled gelatin nanospheres. Scale bars represent $10 \mu \mathrm{m}$.

Video S8 Three-dimensional (3D) video showing the internalization of fluorescently labeled gelatin nanospheres (blue) in the vacuole-like structures of the mCherry fluorescent protein (red) expressing zebrafish macrophage as shown in Video S7 at 24 hours post-intramuscular injection of vancomycin-loaded gelatin nanospheres into a 3-day-old zebrafish larva. This video was converted from a series of the Z-stack images shown in (B) in Figure 7, excluding the green channel images of fluorescent-labeled vancomycin. Scale bars represent $10 \mu \mathrm{m}$.
International Journal of Nanomedicine

\section{Publish your work in this journal}

The International Journal of Nanomedicine is an international, peerreviewed journal focusing on the application of nanotechnology in diagnostics, therapeutics, and drug delivery systems throughout the biomedical field. This journal is indexed on PubMed Central, MedLine, CAS, SciSearch ${ }^{\circledR}$, Current Contents ${ }^{\circledR} /$ Clinical Medicine,

\section{Dovepress}

Journal Citation Reports/Science Edition, EMBase, Scopus and the Elsevier Bibliographic databases. The manuscript management system is completely online and includes a very quick and fair peer-review system, which is all easy to use. Visit http://www.dovepress.com/ testimonials.php to read real quotes from published authors. 